\title{
Boosting the Basic Counting on Distributed Streams
}

\author{
Bojian Xu \\ Department of Computer Science \\ Eastern Washington University, Cheney, WA 99004, U.S.A. \\ bojianxu@ewu.edu
}

\begin{abstract}
We revisit the classic basic counting problem in the distributed streaming model that was studied by Gibbons and Tirthapura (GT). In the solution for maintaining an $(\epsilon, \delta)$-estimate, as what GT's method does, we make the following new contributions: (1) For a bit stream of size $n$, where each bit has a probability at least $\gamma$ to be 1 , we exponentially reduced the average total processing time from GT's $\Theta(n \log (1 / \delta))$ to $O\left(\left(1 /\left(\gamma \epsilon^{2}\right)\right)\left(\log ^{2} n\right) \log (1 / \delta)\right)$, thus providing the first sublinear-time streaming algorithm for this problem. (2) In addition to an overall much faster processing speed, our method provides a new tradeoff that a lower accuracy demand (a larger value for $\epsilon$ ) promises a faster processing speed, whereas GT's processing speed is $\Theta(n \log (1 / \delta))$ in any case and for any $\epsilon$. (3) The worst-case total time cost of our method matches GT's $\Theta(n \log (1 / \delta))$, which is necessary but rarely occurs in our method. (4) The space usage overhead in our method is a lower order term compared with GT's space usage and occurs only $O(\log n)$ times during the stream processing and is too negligible to be detected by the operating system in practice. We further validate these solid theoretical results with experiments on both real-world and synthetic data, showing that our method is faster than GT's by a factor of several to several thousands depending on the stream size and accuracy demands, without any detectable space usage overhead. Our method is based on a faster sampling technique that we design for boosting GT's method and we believe this technique can be of other interest.
\end{abstract}

\section{Introduction}

Advances in modern science and technology have given rise to massive data (or so-called big data). Some of the data naturally arrives as streams. Examples include network data packets passing through a router, environmental data collected by sensor networks, and search requests received by search engines. In many cases, such massive streaming data needs to be monitored in a realtime fashion. Such data process requirements make conventional methods such as storing them in a relational database and issuing SQL queries thereafter infeasible, and thus brings up the phenomenon of data stream processing [21,3]. In data stream processing, the workspace is often orders of magnitude smaller than the stream size, requiring the data be processed in one pass.

However, most streaming algorithms need to look at every data element at least once $[2,16$, $1,20,7,9,8,25,11,4]$ (see [21, 3] for many other example references). In some cases where extremely fast paced streaming data is involved, even a single glance at every stream element can be unaffordable. For example, a typical OC48 link transfers 2.5 Gbits per second and AT\&T backbone networks carry over 15 petabytes of data traffic on an average business day. Deploying a streaming algorithm for monitoring purpose to process every data element in such massive data 
streams is very computationally expensive and can greatly hurt the performance of the system. The goal of sublinear-time algorithms is to solve computational problems without having to look at every input data element. However, in sublinear time algorithm design, the input data is often stored statically $[12,17,23,24]$, meaning we can visit any part of the input data at any time if needed.

In this paper, we demonstrate that designing a sublinear-time algorithm for streaming data is also possible, without losing accuracy guarantee compared with linear-time streaming algorithms. In particular, we proposed the first streaming algorithm for the distributed basic counting problem using time sublinear of the stream size in the average case. To our best knowledge, the best prior result [14] for solving this problem has to visit every stream element at least once and thus needs a time cost at least linear of the stream size in any case.

Distributed basic counting. Alice and Bob (called processors) are processing two geographically distributed bit streams $A=\left\{a_{1}, \ldots, a_{n}\right\}$ and $B=\left\{b_{1}, \ldots, b_{n}\right\}$, respectively and in parallel. In each stream, the $i$ th bit is received and processed before the $j$ th bit, if $i<j$. Upon receiving a query, the referee, who is located on another remote site, wants to know the number of 1-bits in the bit-wise OR of the two bit streams that Alice and Bob have observed and processed.

$$
U(A, B)=\sum_{i=1}^{n}\left(a_{i} \vee b_{i}\right)
$$

where $\mathrm{V}$ is the bit-wise logical OR operator and $n$ is the number of bits that Alice and Bob have both received when the query arrives at the referee. Note that both streams evolve over time and thus the stream size $n$ and the value of $U$ monotonically increase over time. The constraints and challenges in the computation of $U$ are: (1) no direct communication between processors is allowed since there are no direct connection between Alice and Bob, (2) use small workspace on the processors as well as on the referee, and (3) use small communication cost on the links connecting the processors and the referee.

The problem can be generalized to $k$ streams $R_{1}, R_{2}, \ldots, R_{k}$, processed by $k$ processors respectively, for some constant $k \geq 2$. For $j=1,2, \ldots, k$, we write the stream $R_{j}$ in the form of $\left\{r_{j, 1}, r_{j, 2}, \ldots, r_{j, n}\right\}$. Upon receiving a query, the referee wants to know the number of 1-bits in the bit-wise $\mathrm{OR}$ of the $k$ streams:

$$
U\left(R_{1}, R_{2}, \ldots, R_{k}\right)=\sum_{i=1}^{n}\left(r_{1, i} \vee r_{2, i} \vee \ldots \vee r_{k, i}\right)
$$

The same constraints and challenges for the 2 -stream case hold in this general setting. Figure 1 shows the system setting that is assumed in the distributed basic counting. Because our method for the 2-stream case can be easily extended for the general setting, we will focus on the 2-stream case in our presentation. The extension for the general setting will be presented in the end.

We refer readers to [14] for a detailed discussion on the extensive applications of the distributed basic counting in large-scale data aggregation and monitoring.

Prior work. A naive method for the referee to maintain the knowledge of $U$ is to get Alice and Bob to continuously forward their stream elements to the referee. The referee will then simply do a straightforward calculation of $U$ in one pass of the two streams, using $O(\log n)$ bits of workspace 


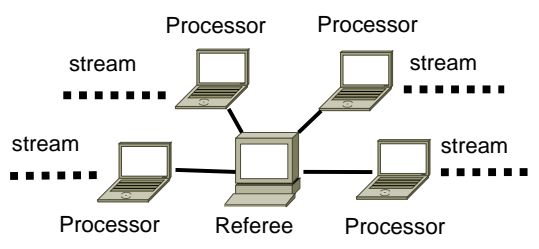

Figure 1: The setting in distributed basic counting

at both the processors and the referee. However, this approach introduces a high communication cost between the processors and the referee, which is prohibited in many applications such as network monitoring. To reduce the communication cost, Gibbons and Tirthapura proposed a communication-efficient distributed computing scheme [14], where Alice and Bob each maintains a small-space data structure (a.k.a. sketch) without communicating neither to each other nor to the referee over the course of stream processing. When the query arrives, the referee will first notify Alice and Bob to send their sketches to the referee. The referee will then retrieve the knowledge of $U$ from the sketches. However, under this distributed computing setting, $\Omega(n)$ bits of communication cost is necessary to get the exact value of $U$ even for randomized algorithms [19]. It is also shown that $\Omega(\sqrt{n})$ bits of workspace is necessary at each processor even for an approximate answer for $U$ with a relative error bound, if Alice and Bob sample their streams independently [14]. In order to achieve a solution of both workspace and communication efficiency, Gibbons and Tirthapura proposed the coordinated adaptive sampling (a.k.a. distinct sampling) technique that uses only $O\left(\left(1 / \epsilon^{2}\right) \log (1 / \delta) \log n\right)$ bits of workspace at each processor and the referee and $O\left(\left(1 / \epsilon^{2}\right) \log (1 / \delta) \log n\right)$ bits of communication cost per link and per query. By using these sublinear (of stream size) space and communication cost, their technique guarantees an $(\epsilon, \delta)$-estimate for $U$ [14]. Their algorithm can be trivially extended to provide an $(\epsilon, \delta)$-estimate of $U$ over multiple streams with the same aforementioned workspace and communication cost.

Definition 1.1. Given the parameters $\epsilon$ and $\delta, 0<\epsilon, \delta<1$, the $(\epsilon, \delta)$-estimate of a nonnegative variable $X$ is a random variable $\hat{X}$, such that:

$$
\operatorname{Pr}[|\hat{X}-X| \leq \epsilon X] \geq 1-\delta
$$

In particular, the $(\epsilon, 0)$-estimate is also called $\epsilon$-estimate.

Definition 1.2 ( $\gamma$-random bit stream.). A bit stream $\left\{a_{1}, a_{2}, \ldots, a_{n}\right\}$ is a $\gamma$-random bit stream, if all the bits in the stream are mutually independent and $\operatorname{Pr}\left[a_{i}=1\right] \geq \gamma$, for $i=1,2, \ldots, n$, where $0<\gamma<1$ is a constant.

The notion of $\gamma$-random bit stream can (roughly) capture the distribution of many real-world bit streams.

\subsection{Our contribution}

We designed a novel sampling technique that enables us to sample the stream without having to check every stream element. By using this fast sampling technique, we are able to boost GT's processing speed in maintaining an $(\epsilon, \delta)$-estimate of the distributed basic counting with negligible extra space usage. Table 1.1 summarizes the performance comparison of our method and GT's. 


\begin{tabular}{|c|c|c|c|}
\hline & GT's [14] & Ours & Note \\
\hline Worst-case total time cost & $\Theta\left(n \log \frac{1}{\delta}\right)$ & $\Theta\left(n \log \frac{1}{\delta}\right)$ & $\begin{array}{l}\text { always occurs with GT's, } \\
\text { but rarely occurs with ours. }\end{array}$ \\
\hline Worst-case per-item time cost & $\Theta\left(\log n \log \frac{1}{\delta}\right)$ & $\Theta\left(\log n \log \frac{1}{\delta}\right)$ & \\
\hline $\begin{array}{l}\text { Avg.-case total time cost for a } \\
\gamma \text {-random bit stream }\end{array}$ & $\Theta\left(n \log \frac{1}{\delta}\right)$ & $O\left(\frac{1}{\gamma \epsilon^{2}} \log ^{2} n \log \frac{1}{\delta}\right)$ & significantly improved \\
\hline $\begin{array}{l}\text { Avg.-case per-item time cost } \\
\text { for a } \gamma \text {-random bit stream }\end{array}$ & $\Theta\left(\log \frac{1}{\delta}\right)$ & $\begin{array}{l}O\left(\frac{1}{n \gamma \epsilon^{2}} \log ^{2} n \log \frac{1}{\delta}\right)=o(1), \\
\text { when } n \text { is large. }\end{array}$ & significantly improved \\
\hline Space cost per-processor (bits) & $O\left(\frac{1}{\epsilon^{2}} \log \frac{1}{\delta} \log n\right)$ & $O\left(\left(\frac{1}{\epsilon^{2}}+\log n\right) \log \frac{1}{\delta} \log n\right)$ & negligible overhead \\
\hline Space cost by the referee (bits) & $O\left(\frac{1}{\epsilon^{2}} \log \frac{1}{\delta} \log n\right)$ & $O\left(\frac{1}{\epsilon^{2}} \log \frac{1}{\delta} \log n\right)$ & \\
\hline Comm. cost per query (bits) & $O\left(\frac{1}{\epsilon^{2}} \log \frac{1}{\delta} \log n\right)$ & $O\left(\frac{1}{\epsilon^{2}} \log \frac{1}{\delta} \log n\right)$ & \\
\hline
\end{tabular}

Table 1: The performance comparison between GT's method and ours.

- The average total processing time for a $\gamma$-random bit stream is reduced from GT's $\Theta\left(n \log \frac{1}{\delta}\right)$ to $O\left(\frac{1}{\gamma \epsilon^{2}} \log ^{2} n \log \frac{1}{\delta}\right)$. Our method not only exponentially improves the overall processing speed, providing the first sublinear-time algorithm in the average case, but also provides a new tradeoff that a lower accuracy demand (a larger value for $\epsilon$ ) promises a faster processing speed, whereas GT's method spends $\Theta\left(n \log \frac{1}{\delta}\right)$ time regardless of the accuracy demand.

- Our method's worst-case total processing time matches GT's $\Theta(n \log (1 / \delta))$, which is necessary. However, this worst-case time cost rarely occurs with our method, whereas GT's method always needs $\Theta(n \log (1 / \delta))$ time in any case.

- Each processor uses $O\left(\left(1 / \epsilon^{2}+\log n\right) \log (1 / \delta) \log n\right)$ bits of workspace in our method. Compared with GT's space usage of $O\left(\left(1 / \epsilon^{2}\right) \log (1 / \delta) \log n\right)$ bits, our method's extra space usage is a lower-order term for any real-world bit stream and a reasonably small $\epsilon$ (say $\epsilon \leq 0.1$ ) and is indeed undetectable in our experiments with both real-world and synthetic data. Further, this extra space cost occurs only $O(\log n)$ times in average during the stream processing.

- The workspace at the referee and the communication cost between the processors and the referee in our method remains the same as GT's.

- We conducted a comprehensive experimental study using both real-world and synthetic data. All experimental results show that our method is faster than GT's by a factor of several to several thousands depending on the stream size and accuracy demand. Our method can potentially save the vast majority of the processing time and energy that is consumed by GT's method in the real-world deployment, where the stream size can be nearly unbounded. All experiments also show that the OS does not detect any extra space cost used by our method compared with GT's.

- The fast sampling technique we proposed can also be of other independent interest in the design of sampling-based algorithms solving other problems.

\subsection{Paper organization}

After a survey of related work, we will present a high-level overview of our method in Section 3, where we will introduce the structure of the coordinated adaptive sampling and show the oppor- 
tunity for boosting. We will then introduce the new sampling technique for boosting in Section 4. By plugging the faster sampling technique into the coordinated adaptive sampling, we are able to present the complete picture of our method in Section 5. The details of a comprehensive experimental study using both real-world and synthetic data are given in Section 6. This paper is concluded by Section 7 .

\section{Related work}

In this section, we summarize the results on basic counting in the streaming model under various constraints and settings. For a broader overview of stream processing, we refer readers to the surveys $[21,3]$.

While the small-space basic counting of a whole single stream is trivial, it becomes much harder when counting the number of 1-bits on the union of multiple geographically distributed streams. It is shown in [19] that $\Omega(n)$ bits of workspace is necessary for an exact answer even for randomized algorithms, where $n$ is the size of each single stream. Datar et al. [11] considered the basic counting on a single stream, given the constraint of sliding windows. It is easy to show $\Omega(N)$ bits is required to maintain the exact knowledge of how many 1-bits in a sliding window of size $N$, representing the most recently received $N$ bits. They further showed a space lower bound of $\Omega\left((1 / \epsilon) \log ^{2}(\epsilon N)\right)$ bits for both deterministic and randomized algorithms for maintaining an $\epsilon$-estimate of the basic counting over a sliding window of size $N$. They also proposed an exponential histogram based deterministic algorithm that guarantees an $\epsilon$-estimate using workspace matching the above space lower bound, but their algorithm cannot work for the union of multiple bit streams under the sliding windows model.

Gibbons and Tirthapura [15] solved this new challenge by extending and applying their technique from [14] to the sliding window setting over multiple streams. Instead of maintaining one sample as was done in [14] for the case without sliding window, they maintain $\log N^{1}$ samples where every sample has a different sample probability from $1,1 / 2, \ldots, 1 /\left(2^{\log N}\right)$, because the number of 1-bits in the sliding window can vary over time depending upon the input stream and thus one fixed good sample probability cannot be predetermined beforehand. By maintaining multiple samples, their technique is able to pick the best samples with the right sample probabilities from the multiple processors at the query time, so that the referee is guaranteed to have a good estimate for the basic counting on the union of the bit streams over the sliding window.

$\mathrm{Xu}$ et al. [26] considered the sliding window based basic counting on an asynchronous stream, where the arrival order of the stream elements is not necessarily the same as the order at which they were created. Such an asynchronous stream model is motivated by the real-world scenarios such as network data packets being received out of order at the destination due to the network delay and multi-path routing. The core idea of their solution is mostly identical to the one from [15] but is modified for asynchronous streams. It is also easy to extend their solution so that it can work for the union of multiple streams. Busch and Tirthapura [5] later also solved the asynchronous stream basic counting problem over sliding windows for one stream. Their solution is deterministic and is based on a novel data structure called splittable histogram, but it is not clear how to extend their method to multiple streams.

The coordinated random sampling technique [14] can also be used for counting $F_{0}[2,4,18]$, the number of distinct elements, over one or the union of multiple data streams, with or without

\footnotetext{
${ }^{1}$ In this paper, we use the convention that, unless specified explicitly, the base of logarithm function is 2 .
} 
sliding windows $[14,15,13]$. Pavan and Tirthapura [22] generalized the data stream model in the calculation for $F_{0}$. In their stream model, every stream element is no longer a single integer but is a range of continuous integers. The $F_{0}$ is defined as the number of distinct integers in the union of the ranges that have been received in the stream. A trivial solution is to expand each range into a sequence of integers and use an existing $F_{0}$ algorithm to process each integer. The time cost for processing a range will then be at least linear of the range size, which is not acceptable when the range size is large. They proposed a divide and conquer based strategy, such that the time cost for processing each range is only a logarithm of the range size. Part of the idea behind our new sampling technique presented in this paper is inspired by their work.

All these related work need to observe each stream element at least once, leading to their time costs to be at least linear of the stream size.

\section{A high-level overview}

The high-level structure of our method is the coordinated adaptive sampling by Gibbon and Tirthapura [14], but uses a different hash function for the random sampling. We exploit the properties of the hash function, so that we can do random sampling over the data stream without having to check every stream element.

\subsection{Coordinated adaptive random sampling}

Alice and Bob use the same sampling procedure and the sample size, so we will only describe the behavior of Alice.

Random sampling. Alice maintains a sample of some known size $\alpha$, which will be determined later. She randomly selects each 1-bit (by storing the bit's stream location index) into the sample with some probability $p$. After processing the stream, the number of 1-bits selected into the sample multiplied by $1 / p$ can be a good estimate of the number of 1-bits in the stream, if $\alpha$ is large enough.

Adaptive random sampling. However, Alice does not know in advance how many 1-bits will be present in her stream and thus cannot decide an appropriate sample probability $p$. If the sample probability is too high, the sample size may not be big enough to accommodate all the selected 1-bits; if the sample probability is too small, the sample may not select enough 1-bits to yield a good estimate. To overcome this difficulty, Alice adaptively changes the sample probability over the course of her stream processing. The sample probability is determined by the sample level $\ell$, which starts from 0 and increases towards $1,2, \ldots$. At sample level $\ell$, every incoming 1-bit is selected into the sample with sample probability $P_{\ell}=1 / 2^{\ell}$ (In our method, $P_{\ell}$ is not exactly but is nearly equal to $1 / 2^{\ell}$ and will be clear later). Alice is always aware of her current sample probability by remembering her current sample level $\ell$.

Coordinated adaptive random sampling. However, Gibbons and Tirthapura showed that if all the processors do their random sampling independently, $\Omega(\sqrt{n})$ bits of workspace at each processor is necessary even for an estimate of the distributed basic counting with a relative error bound [14]. The way in [14] to overcome this space lower bound is to coordinate the random sampling procedures by using a common hash function to simulate the sampling procedure. The hash function used in [14] is pair-wise independent and is defined over the field $G F\left(2^{m}\right)$, where $m=\log n$. Their hash function maps the stream location indexes $\{1,2, \ldots, n\}$ to the sample levels $\{0,1, \ldots, m\}$. The hash function provides that the probability that a stream location index 
is hashed to a particular sample level $i$ is exactly equal to $1 / 2^{i+1}$. An incoming 1-bit will be selected into the sample if and only if its stream location index is hashed to a sample level $i \geq \ell$, where $\ell$ is the current sample level. Thus, in average, only $1 / 2^{\ell+1}+1 / 2^{\ell+2}+\ldots+1 / 2^{m} \approx 1 / 2^{\ell}$ of the $n$ stream element locations will be selected, which is the goal of the adaptive sampling each stream location should be selected with probability $P_{\ell}=1 / 2^{\ell}$. Clearly, coordinated adaptive random sampling yields the same set of selected stream locations at all processors if they are on the same sample level. Of course, those selected locations that do not have 1-bits will not be saved in the sample.

When the sample is full. The sample level will be incremented by one, when the sample becomes full. All the 1-bits that are currently in sample and whose hash values are less than the new sample level $\ell$ will be discarded from the sample. By doing so, all the 1-bits that Alice has received so far have been assigned the same sample probability $P_{\ell}$ for selection. Alice will then continue her processing of the new incoming 1-bits using the current sample probability $P_{\ell}$ until the sample becomes full again.

When the referee receives a query for $U$. All processors will send their samples and sample levels to the referee. For each sample, if its sample level is smaller than $\ell_{\text {max }}$, the largest sample level the referee has received, the referee will increase its sample level to $\ell_{\max }$ by retaining only those 1-bits whose hash values are not less than $\ell_{\max }$. After this resampling step, all the samples will share the same sample level. The referee will then union all the samples by conducting bit-wise OR of the selected 1-bits according to their stream locations. The number of 1-bits in union multiplied by $1 / P_{\ell_{\max }}$ will be returned by the referee as the answer to the query for $U$.

\subsection{Use a different hash function for sampling}

Our method follows GT's structure but uses a different hash function to conduct the coordinated adaptive sampling procedure. Since the choice of the hash function is critical for the improvement of the processing speed, we provide its details in the following. We first pick a prime number $p$ uniformly at random from $[10 n, 20 n]$, then pick another two numbers $a$ and $b$ from $\{0,1, \ldots, p-1\}$ and $a \neq 0$. The hash function $h:\{1,2, \ldots, n\} \rightarrow\{0,1, \ldots, p-1\}$ is defined as: $h(x)=(a \cdot x+b)$ $\bmod p$. It is well known [6] that:

- $h(x)$ is uniformly distributed in $\{0,1, \ldots, p-1\}$.

- $h$ is pair-wise independent, i.e., for any $x_{1} \neq x_{2}$ and $y_{1}, y_{2}: \operatorname{Pr}\left[\left(h\left(x_{1}\right)=y_{1}\right) \wedge\left(h\left(x_{2}\right)=y_{2}\right)\right]=$ $\operatorname{Pr}\left[h\left(x_{1}\right)=y_{1}\right] \cdot \operatorname{Pr}\left[h\left(x_{2}\right)=y_{2}\right]$.

For each $\ell \in\{0,1, \ldots,\lfloor\log \mathrm{p}\rfloor\}$, we define:

$$
R_{\ell}=\left\{0,1, \ldots,\left\lfloor p / 2^{\ell}\right\rfloor-1\right\}
$$

Our sampling policy is that a stream element $a_{i}$ will be selected into the sample if $a_{i}=1$ and $h(i) \in R_{\ell}$. Since the hash values $\{0,1, \ldots, p-1\}$ are uniformly distributed, the probability of selecting any 1-bit at sample level $\ell$ is:

$$
P_{\ell}=\left|R_{\ell}\right| / p=\left\lfloor p / 2^{\ell}\right\rfloor / p \approx 1 / 2^{\ell}
$$

Algo. 1 shows the pseudocode of GT's coordinated adaptive sampling, plugged in our new hash function, for estimating $U$ of two streams. 


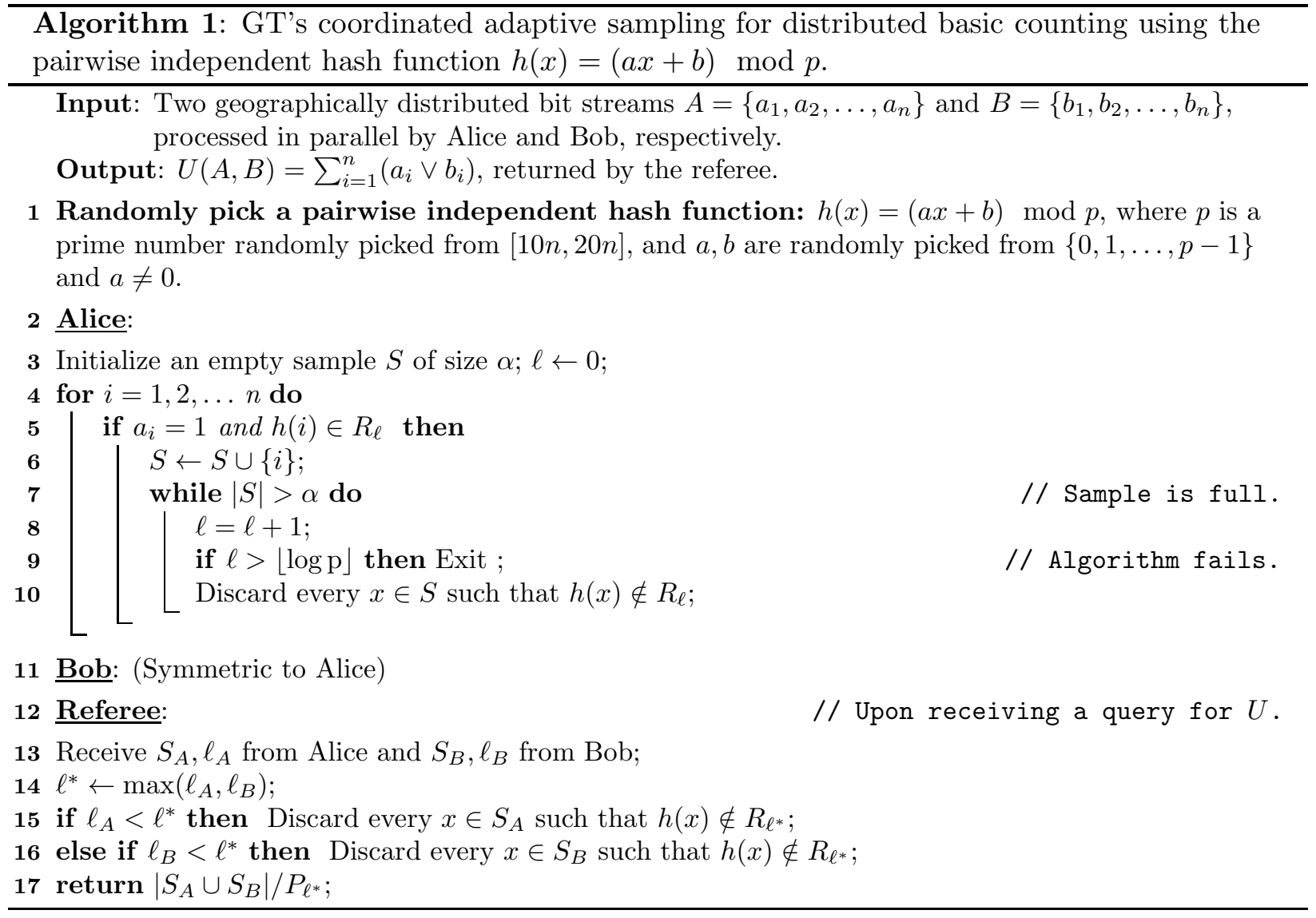

\subsection{The opportunity for speedup}

GT's method checks every stream element at least once (Line 4-5, Algo. 1), which yields their time cost for processing a stream of $n$ bits is at least $\Theta(n)$. We observe that the hash values $h(1), h(2), \ldots$ of the hash function $h$ we use (Section 3.2) follows some pattern, which will be made clear later. By taking advantage of this pattern, it is possible to skip over some stream elements without checking their hash values, because their hash values can be proved to be out of $R_{\ell}$. In particular, suppose $a_{i}$ is the element that we are currently processing and $a_{i+d}$ is the next element whose hash value is within $R_{\ell}$, we are able to design an algorithm that finds the value of $d$ using $O(\log d)$ time. That says we will not need to literally check the elements $a_{i+1}, a_{i+2}, \ldots, a_{a+d-1}$ one by one. Our algorithm for finding the value of $d$ is based on a recursive decomposition of the task, so that the new instance of the problem has a significantly smaller problem size. The recursive decomposition is based some properties of the hash function $h(x)=(a \cdot x+b) \bmod p$ that we use for coordinated adaptive sampling.

\section{The new technique: direct sampling}

The challenge for fast sampling is to find the next stream location that will be selected into the sample if that location has a 1-bit, without having to check the hash value of every stream location up to that sampled location. That is, suppose we are now at sample level $\ell$ and at the stream 
location $x$, we want to compute the following function quickly:

$$
\operatorname{DirectSample}(x, \ell, p, a, b)=x+\mathcal{N}_{x}^{\ell}
$$

where

$$
\mathcal{N}_{x}^{\ell}=\min \left\{i \geq 0 \mid h(x+i)=(a(x+i)+b) \quad \bmod p \in R_{\ell}\right\}
$$

Note that for an arbitrary setting of the parameters of $h, \mathcal{N}_{x}^{\ell}$ may not be well defined because the set $\left\{i \geq 0 \mid h(x+i) \in R_{\ell}\right\}$ can be empty. For example, if $p=8, a=4, b=2, \ell=2, x=1$, then for any $i \geq 0$, the value of $h(x+i)$ is always either 2 or 6 , neither of which belongs to $R_{\ell}=\{0,1\}$. However, it can be shown that when $p$ is a prime number, $\mathcal{N}_{x}^{\ell}$ is always well defined. We first prove that $\{h(x), h(x+1), \ldots h(x+p-1)\}$ and $\{0,1, \ldots, p-1\}$ are actually the same set of $p$ distinct numbers.

Lemma 4.1. For any $p>0,0<a<p, 0 \leq b<p$, and $x \geq 1$, if $p$ is a prime number, then:

$$
\{h(x), h(x+1), \ldots h(x+p-1)\}=\{0,1, \ldots, p-1\}
$$

Proof. We prove the lemma by contradiction. Let $A$ denote $\{h(x), h(x+1), \ldots h(x+p-1)\}$ and B denote $\{0,1, \ldots, p-1\}$. Suppose $A \neq B$, then since every $h(x+k)$ for $k=0,1, \ldots, p-1$ is a member of $B$ and $|A|=|B|$, there must exist two integers $i$ and $j$, such that $0 \leq i<j \leq p-1$ and $h(x+i)=h(x+j)$. That is,

$$
\begin{aligned}
& a(x+i)+b=a(x+j)+b \bmod p \\
\Longleftrightarrow & a i=a j \bmod p \\
\Longleftrightarrow & a(j-i)=0 \bmod p
\end{aligned}
$$

Combining the fact that $0<a<p$ and $0<j-i<p$, the above Equation 2 indicates that $p$ is not a prime number, which is a contradiction, thus the lemma is proved.

Lemma 4.2. For any $p>0,0<a<p, 0 \leq b<p, x \geq 1$, and $L \geq 0$, if $p$ is a prime number, then $\{i \geq 0 \mid h(x+i) \leq L\}$ is always not empty.

Proof. By Lemma 4.1, we know that $\{h(x), h(x+1), \ldots, h(x+p-1)\}=\{0,1, \ldots, p-1\}$. Because $L \geq 0$, there must exist at least one member in the set $\{h(x), h(x+1), \ldots, h(x+p-1)\}$ whose value is not larger than $L$, so the lemma is proved.

Lemma 4.3. $\mathcal{N}_{x}^{\ell}$ always exists.

Proof. Recall that by design the sample level $\ell$ is no more than $\lfloor\log \mathrm{p}\rfloor$ (Line 9, Algo. 1), so $\left\lfloor 2^{-\ell} p\right\rfloor-1 \geq 0$ is always true. By setting $L=\left\lfloor 2^{-\ell} p\right\rfloor-1 \geq 0$, Lemma 4.2 has proved that the set $\{i \geq 0 \mid h(x+i) \leq L\}$ is always not empty, so $\mathcal{N}_{x}^{\ell}=\min \left\{i \geq 0 \mid h(x+i) \in R_{\ell}\right\}=\min \{i \geq 0 \mid$ $\left.h(x+i) \leq\left\lfloor 2^{-\ell} p\right\rfloor-1\right\}$ is always well defined and exists.

We can now focus on the design of an efficient algorithm for finding $\mathcal{N}_{x}^{\ell}$.

Problem 1. Given integers $p>0,0 \leq a<p, 0 \leq u<p$, and $L \geq 0$, computer the following $d$ :

$$
d=\left\{\begin{array}{l}
\min \{i \geq 0 \mid(u+i \cdot a) \quad \bmod p \leq L\} \\
\quad \text { if }\{i \geq 0 \mid(u+i \cdot a) \bmod p \leq L\} \neq \emptyset \\
-1, \quad \text { otherwise }
\end{array}\right.
$$


Let $Z_{p}$ denote the ring of the nonnegative numbers modulo $p$. Observe that the sequence of the values of $h(x+i)$, for $i=0,1,2, \ldots$, is an arithmetic progression over $Z_{p}$ with common difference a. The task of finding $\mathcal{N}_{x}^{\ell}$ is reduced to finding $d\left(=\mathcal{N}_{x}^{\ell}\right)$ in Problem 1 by setting:

$$
u=h(x)=(a x+b) \quad \bmod p, \quad L=\left\lfloor 2^{-\ell} p\right\rfloor-1
$$

Let $S=\langle u \bmod p,(u+a) \bmod p,(u+2 a) \bmod p, \ldots\rangle$. For $i=0,1, \ldots$, let $S[i]=(u+i \cdot a)$ $\bmod p$, the $i$ th element in the sequence $S$. Problem 1 can be restated as follows: find the smallest $i \geq 0$, such that $S[i] \leq L$, or report that such $i$ does not exist.

\subsection{Possible solutions.}

A naive method, as used in [14], is to iteratively check the value of $(u+i \cdot a) \bmod p$ for $i=0,1, \ldots$ It takes $O(d)$ time if a nonnegative $d$ is eventually found and the procedure will even never stop if a nonnegative $d$ does not exist at all.

A better solution is to use the Hits function from [22]. Given a fixed-sized prefix of the sequence $S$, Hits can efficiently calculate the number of elements in the prefix whose values are less than or equal to $L$ using $O(\log y)$ time, where $y$ is the length of the prefix. Assuming a nonnegative $d$ exists for Problem 1, if we can make a good guess of the length of the prefix, such that $y \geq d$, meaning the prefix includes at least one element whose value is less than or equal to $L$. Then we can use a binary search over the prefix to locate the first element whose value is less than or equal to $L$. Altogether, it will take $O\left(\log ^{2} y\right)$ time, because there are $O(\log y)$ binary search steps and each binary search step takes $O(\log y)$ time for Hits. However, this method has several unsolved issues: (1) It is not clear how to make a good guess of the length of the prefix so that $y \geq d$, since $d$ is unknown: If $y \gg d$, it wastes computational time; If $y<d$, we will have to guess a longer prefix of $S$, leading to an inefficient procedure. (2) Even if a good guess of $y$ is made, it will take $O\left(\log ^{2} y\right)$ time to locate the first element whose value is less than or equal to $L$. We look for a solution that takes only $O(\log y)$ of time. (3) If $d \geq 0$ does not exist, the binary search method of using Hits fails.

Another possible solution is to use the MinHit function from [10]. Given a prefix of size $y$ of the sequence $S$, by using $O(\log y)$ of time, MinHit can find the first element in the prefix that is less than or equal to $L$ or return -1 if such element does not exist. Similar unsolved issues exist: (1) It is not clear how to make a good guess of the size of a prefix so that it includes at least one element that is not larger than $L$. Bad guesses lead to wasting of computational time as we have explained in the possible solution using Hits. (2) If $d \geq 0$ does not exist, the method of using MinHit fails.

\subsection{Our solution}

We now present our algorithm called NextHit for solving Problem 1. Our approach is to directly modify the internal mechanism of the MinHit function so it can work with an infinitely long sequence.

Note that the sequence $S$ is an arithmetic progression over $Z_{p}$ with common distance $a$. For $i=0,1, \ldots$, let $S_{i}$ denote the subsequence which is the $i$ th round of the progression and $f_{i}$ denote the first element in $S_{i}$. Let $F=\left\langle f_{0}, f_{1}, f_{2}, \ldots\right\rangle$ and $\left|S_{i}\right|$ denote the number of elements in $S_{i}$. Figure 4.1 shows an example $S$ and its $S_{i}$ subsequences and $F$ sequence.

We start the design of NextHit with the following critical observation from [22]. 


\begin{tabular}{r|cc|ccc|ccc|cccc|ccc|c}
$i$ & 0 & 1 & 2 & 3 & 4 & 5 & 6 & 7 & 8 & 9 & 10 & 11 & 12 & 13 & 14 & $\cdots$ \\
\hline \hline$S[i]$ & $\mathbf{7}$ & 11 & $\mathbf{2}$ & 6 & 10 & $\mathbf{1}$ & 5 & 9 & $\mathbf{0}$ & 4 & 8 & 12 & $\mathbf{3}$ & 7 & 11 & $\cdots$ \\
\hline & $f_{0}$ & $f_{1}$ & & & $f_{2}$ & & & $f_{3}$ & & & $f_{4}$ & & & $\cdots$ \\
\cline { 2 - 11 } & $S_{0}$ & & $S_{1}$ & & & $S_{2}$ & & & \multicolumn{3}{|c}{$S_{3}$} & & & $S_{4}$ & & $\cdots$
\end{tabular}

Figure 2: An example sequence $S=\langle u+0 \cdot a \bmod p,(u+1 \cdot a) \bmod p,(u+2 \cdot a) \bmod p, \ldots\rangle$, where $u=7, a=4, p=13$. For $i=0,1, \ldots$ : (1) $S[i]$ denotes the $i$ th element in $S$; (2) The subsequence $S_{i}$ denotes the $i$ th round of the progression over $Z_{p} ;(3) f_{i}$ is the smallest element in $S_{i}$. Suppose $L=1$ in the setting of Problem 1, the answer should be $d=5$, because $S[5]$ is the first element whose value is not larger than $L$.

Observation 4.1 (Observation 2 of [22]). Sequence $\bar{F}=F \backslash\left\{f_{0}\right\}=\left\langle f_{1}, f_{2}, \ldots\right\rangle$ is an arithmetic progression over $Z_{a}$, with common difference $a-r$ (or $-r$, equivalently), where $r=p$ mod $a$. That $i s$, for every $i \geq 1$ :

$$
f_{i}=\left(f_{1}+(i-1) \cdot(a-r)\right) \bmod a
$$

Figure 4.1 shows an example $\bar{F}$ sequence.

The next lemma says that if $\{i \geq 0 \mid(u+i \cdot a) \bmod p \leq L\} \neq \emptyset, S[d]$ must be the first element whose value is not larger than $L$ in the sequence $F$.

Lemma 4.4 (A generalization of Lemma $3.6[10]$ ). If $d \neq-1, S[d]=f_{m} \in F$, where $m=\min \{i \geq$ $\left.0 \mid f_{i} \leq L\right\}$.

Proof. First, we prove $S[d] \in F$. Suppose $S[d] \notin F$ and $S[d] \in S_{t}$, for some $t$. Let $f_{t}=S\left[d^{\prime}\right]$, so $d^{\prime}<d$. Since $S[d]$ and $f_{t}$ both belong to $S_{t}$ while $S[d]$ is not the first element of $S_{t}$, we have $f_{t} \leq S[d] \leq L$. Because $d^{\prime}<d$, if $d^{\prime}$ is not returned, $d$ will not be returned either. This yields a contradiction. Next, we prove $S[d]=f_{m}$. Suppose $S[d]=f_{m^{\prime}}$, where $m^{\prime}>m$. Let $f_{m}=S\left[d^{\prime}\right]$. Note that $d^{\prime}<d$ because $m<m^{\prime}$. Since $d^{\prime}<d$ and $S\left[d^{\prime}\right] \leq L$, if $d^{\prime}$ is not returned, $d$ will not be returned either. This is also a contradiction.

By observing $S$ is an arithmetic progression over $Z_{p}$, it is easy to get the following lemma.

Lemma 4.5 (Lemma 3.7 of $[10])$. If $m=\min \left\{i \geq 0 \mid f_{i} \leq L\right\}$ exists, then $d=\left(m p-f_{0}+f_{m}\right) / a$.

\subsubsection{The overall strategy for solving Problem 1}

We will first find the value of $m$, which will give us the value of $f_{m}$ due to Equation 3 . Then, we will get the value of $d$ (thus solving Problem 1) by using Lemma 4.5. That is, the task of solving Problem 1 can be reduced to the task of finding $m$, which in fact, as we will explain soon, is another instance of Problem 1 with a different parameter setting.

The next lemma will be used to convert an instance of Problem 1 to another instance of Problem 1 which has a different set of parameters but returns the same answer.

Lemma 4.6 (A generalization of Lemma $3.8[10]$ ). Let $d$ denote the answer to an instance of Problem 1 with parameter setting: $p, a, u, L$. Let $d^{\prime}$ denote the answer to another instance of Problem 1 with parameter setting: $p^{\prime}=p, a^{\prime}=p-a, u^{\prime}=(p-u+L) \bmod p, L^{\prime}=L$. Then: $d=d^{\prime}$ 
Proof. Let $P=\{i \geq 0 \mid(u+i \cdot a) \bmod p \leq L\}$ and $P^{\prime}=\{j \geq 0 \mid((p-u+L) \bmod p+j \cdot(p-a))$ $\bmod p \leq L\}$. In the trivial case where $P$ and $P^{\prime}$ are both empty, clearly $d=d^{\prime}=-1$. In the nontrivial case where $P$ and $P^{\prime}$ are not both empty, we first prove $P=P^{\prime}$ by showing $P \subseteq P^{\prime}$ and $P^{\prime} \subseteq P$.

1. $P \subseteq P^{\prime}$. Suppose $\gamma \in P$, then $\gamma \geq 0$ and $(u+\gamma \cdot a) \bmod p \leq L$. We want to prove $\gamma \in P^{\prime}$.

$$
\begin{aligned}
& {[(p-u+L) \bmod p+\gamma \cdot(p-a)] \bmod p} \\
& =[p-u+L+\gamma \cdot(p-a)] \bmod p \\
& =[L-(u+\gamma \cdot a)] \bmod p \\
& =[L-(u+\gamma \cdot a) \bmod p] \bmod p \leq L
\end{aligned}
$$

The inequality is due to the fact that $0 \leq(u+\gamma \cdot a) \bmod p \leq L$. So, $\gamma \in P^{\prime}$.

2. $P^{\prime} \subseteq P$. Suppose $\gamma \in P^{\prime}$, then $\gamma \geq 0$ and $[(p-u+L) \bmod p+\gamma \cdot(p-a)] \bmod p \leq L$. We want to prove $\gamma \in P$.

$$
\begin{aligned}
& {[(p-u+L) \quad \bmod p+\gamma \cdot(p-a)] \quad \bmod p } \\
= & {[L-(u+\gamma \cdot a) \bmod p] \quad \bmod p \leq L }
\end{aligned}
$$

If $(u+\gamma \cdot a) \bmod p>L$, say $(u+\gamma \cdot a) \bmod p=L+\sigma<P$ for some $\sigma>0$. From the above inequality, we can have that $(-\sigma) \bmod p=p-\sigma \leq L$, i.e., $L+\sigma \geq P$, which yields a contradiction. So, $(u+\gamma \cdot a) \bmod p \leq L$, i.e., $\gamma \in P$.

Because $d$ and $d^{\prime}$ are the smallest values in $P$ and $P^{\prime}$ respectively, the fact $P=P^{\prime} \neq \emptyset$ directly yields $d=d^{\prime}$.

\subsubsection{The mechanism of NextHit}

Now we are ready to design the algorithmic mechanism of the NextHit algorithm using the discoveries we have presented. Given an instance of Problem 1 with parameter setting $p, a, u, L$, the easy case is $u \leq L$, for which we will obviously return $d=0$. Otherwise, because $S[d]=f_{m}$ (Lemma 4.4), we will reduce the task of solving Problem 1 with parameter setting $p, a, u, L$ to the task of finding $m$ such that $f_{m}$ is the first element whose value is not larger than $L$ in the $\bar{F}$ sequence. Observe that the $\bar{F}$ sequence is also an arithmetic progression over a smaller ring $Z_{a}$ with common distance $a-r$ (Observation 4.1), so the task of finding $m$ is actually a new instance of Problem 1 with the following parameter setting:

$$
p_{\text {new }}=a, \quad a_{\text {new }}=a-r, \quad u_{\text {new }}=f_{1}, \quad L_{\text {new }}=L
$$

Note that the returned value of the above instance actually will be equal to $m-1$, because the $\bar{F}$ sequence is one indexing based. After $m$ is recursively calculated, we will use Observation 4.1 to directly obtain $f_{m}$ :

$$
f_{m}=\left(f_{1}+(m-1)(a-r)\right) \bmod a
$$

Then we can use Lemma 4.5 to directly calculate $d$, which is the answer to the original instance of Problem 1:

$$
d=\left(m p-f_{0}+f_{m}\right) / a
$$




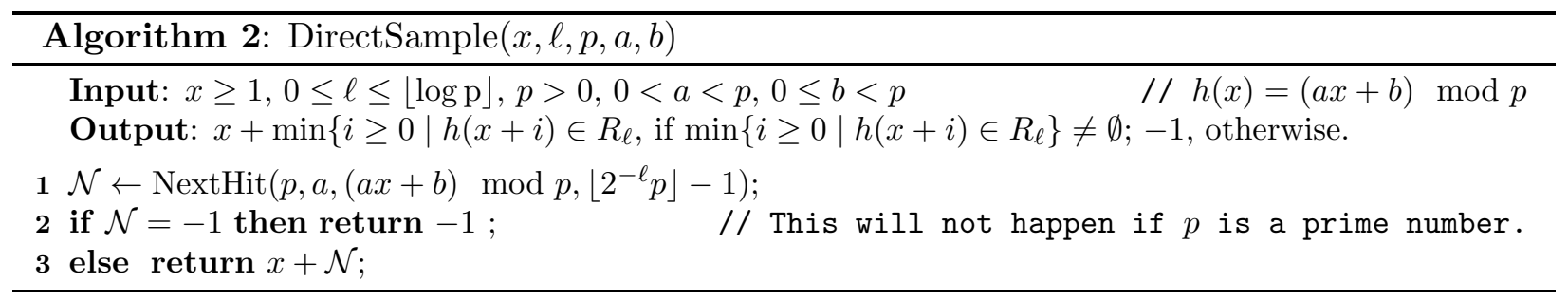

However, the recursion (Equations 4) may not always be effective because the new progression's common distance $a-r$ may not be much smaller than the old progression's common distance $a$ (We will explain later why the size of the common distance is relevant to both the time and space complexities of the NextHit algorithm). Fortunately, by using Lemma 4.6, we can overcome this difficulty by converting the recursive instance to another instance which returns the same answer but works on a progression with a smaller common distance. We summarize the two possible reductions in the following.

Case 1: $a-r \leq a / 2$. We want to work with $a-r$. Problem 1 is recursively reduced to a new instance of Problem 1 of a smaller size that finds $m$ over sequence $\bar{F}$ by setting:

$$
p_{\text {new }}=a, \quad a_{\text {new }}=a-r, \quad u_{\text {new }}=f_{1}, \quad L_{\text {new }}=L
$$

Case 2: $r<a / 2$. We want to work with $r$. We first recursively reduce Problem 1 to the same setting as in Case 1, which will be further converted to the following parameter setting because of Lemma 4.6:

$$
\begin{gathered}
p_{\text {new }}=a, \quad \begin{array}{c}
a_{\text {new }}=a-r, \quad u_{\text {new }}=f_{1}, \quad L_{\text {new }}=L \\
\Downarrow(\text { Lemma 4.6) }
\end{array} \\
p_{\text {new }}=a, a_{\text {new }}=r, u_{\text {new }}=\left(a-f_{1}+L\right) \bmod a, L_{\text {new }}=L
\end{gathered}
$$

After adding some trivial recursion exit conditions, we present the pseudocode of NextHit in Algo. 3, which directly reflects the algorithmic idea that we have presented. Once NextHit is clear, the calculation of DirectSample becomes trivial and is presented in Algo. 2.

Theorem 4.1 (Correctness and time and space complexity of NextHit). $\operatorname{NextHit}(p, a, u, L)$ solves Problem 1 using $O(\log a)$ time and $O(\log p \cdot \log a)$ bits of space. When $p$ is a prime number, $\operatorname{NextHit}(p, a, u, L)$ solves Problem 1 using $O(\min (\log a, \log d))$ time and $O(\log p \cdot \min (\log a, \log d))$ bits of space, where $d$ is the value returned by NextHit.

Proof. Correctness. Recall that $\operatorname{NextHit}(p, a, u, L)$ should return $d=\min \{i \geq 0 \mid(u+i \cdot a)$ $\bmod p \leq L\}$, if such $d$ exists; otherwise, it will return $d=-1$. The three exit conditions (Line 1-3) capture all the possible cases where the algorithm can return and exit directly. At the end of Line 3, we know $S[d]$ does not occur in $S_{0}$ and $p>a \geq 2$, so we are ready to reduce the task of finding $d$ to the task of finding $m$ over the sequence $\bar{F}$. Line $4-7$ correctly calculate $f_{1}$ and $r$ as the preparation work for the subsequent recursive calls at Line $8-9$. Line 8 calculates the 0 -based index of the first element in the sequence $\bar{F}$ that is not larger than $L$. By Lemma 4.6, we know the recursive call at Line 9 will return the same result as that from Line 8 . In the case where -1 is returned by the recursive call (Line 8 or 9), it means no element in the $\bar{F}$ sequence is less than or equal to $L$. In that case, we will return $d=-1$ (Line 10). Otherwise, Line 12-13 calculates and returns the value of $d$ using the results returned by the preceding recursive call (Lemma 4.5). 


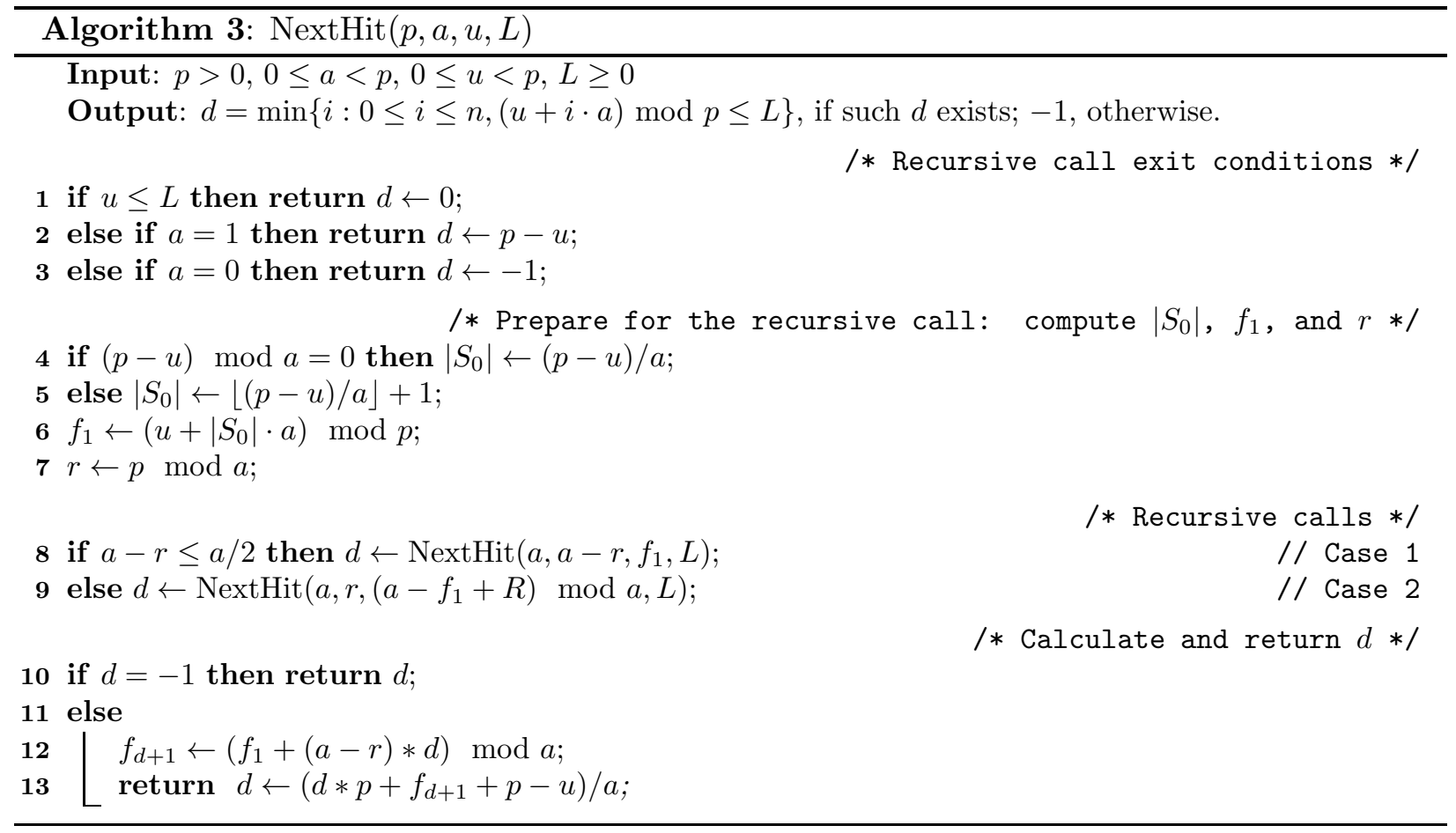

Time Complexity. We assume that the additions, multiplications, and divisions take unit time. At each recursive call (Line 8 or 9 ), we have $a_{n e w} \leq a / 2$, plus the fact that in the worst case the recursion will return when $a=1$ (Line 2 ), so the depth of the recursions is no more than $\log a$. Because the time cost for the local computation in each recursive call is constant (Line $1-7$ and $10-13)$, the time complexity of NextHit is $O(\log a)$.

In the particular case where $p$ is a prime number, which is the case in the use of NextHit for distributed basic counting in this paper, we know the value of $d$ returned by NextHit is always non-negative (Lemma 4.2). In this case, the length of the arithmetic progression that the caller of NextHit works with is $d+1$. Because at each recursive call, we have the common distance in the new progression reduced by at least half, so the length of the progression that the next recursive NextHit will work with is no more than a half of the caller's progression. This observation implies that the depth of the recursion is no more than $\log d$, so the overall time cost is bounded by $O(\log d)$. Comparing with the time cost of NextHit in the general case, we see the time cost of NextHit in this particular case is $O(\min (\log a, \log d))$.

Space Complexity. In each recursive call, NextHit needs to store a constant number of local variables such as $p, a, u, L$, etc. Since $p$ dominates $a, u$ and $L$ (if $L \geq p$, then NextHit() returns without recursive calls (Line 1$)$.), each recursive call needs $O(\log p)$ stack space. Since the depth of the recursion is no more than $O(\log a)$, which we have explained in the time complexity analysis, the space cost of the NextHit algorithm is upper bounded by $O\left(\log ^{2} p\right)$ bits.

In the case where $p$ is a prime number, the depth of the recursion is bounded by $O(\log d)$, which we have explained the time complexity analysis, so the total space cost is no more than $O(\log p \cdot \log d)$. Comparing with the space cost of NextHit in the general case, we get the time cost of NextHit in this particular case is $O(\log p \cdot \min (\log a, \log d))$. 
Corollary 4.1 (Correctness and time and space complexity of DirectSample). DirectSample $(x, \ell, p, a, b)$ finds the next $\ell$-level sample location on or after the $x$ th stream element using $O(\min (\log a, \log d))$ time and $O(\log n \cdot \min (\log a, \log d))$ bits of space, where $n$ is an upper bound of the stream size and $x+d$ is the value returned by DirectSample.

Proof. The time and space cost of DirectSample is dominated by the NextHit subroutine. By Theorem 4.1 and combining the fact that $p$ is a random prime number chosen from $[10 n, 20 n]$, we can get the claim proved.

\section{Boosting the distributed basic counting via direct sampling}

Now we present the complete picture of our new method for basic counting on the union of multiple streams, followed by its correctness proof and the analysis of its time, space, and communication complexities.

\subsection{Algorithm description}

The formal description of the algorithm is presented in Algo. 4. Note that one instance of the algorithm does not automatically yield an $(\epsilon, \delta)$-estimate of $U$, but produces an estimate within a factor of $\epsilon$ of $U$ with a constant probability. The standard technique to reduce the failure probability from a constant to the user-input parameter $\delta$ is to run in parallel $O(\log (1 / \delta))$ independent instances of the algorithm and return the median of the results from those instances.

The overall structure of the algorithm is still coordinated adaptive sampling, but uses the hash function $h$ and thus can use the direct sampling technique as a subroutine for a faster sampling procedure. We first randomly pick a pairwise independent hash function $h$ (Line 1) as defined in Section 3.2. The hash function $h$ is shared by both the stream processors (Alice and Bob) and the referee for coordinated sampling. Each processor starts with an empty sample of size $\alpha=\left\lceil 60 / \epsilon^{2}\right\rceil$ and the sample level $\ell=0$ (Line 3).

The use of direct sampling. The algorithm does not check the hash value of every stream element. After processing a particular stream element $a_{i}$ (Line 6-11), the algorithm calls DirectSample $(i+$ $1, \ell, p, a, b)($ Line 12) to find the next stream element that needs to be checked at the current sample level $\ell$. The algorithm will go to sleep until that element arrives. That is, all the stream elements that do not have the possibility to be selected regardless of its value will be ignored (Line 5). When the element whose location index was returned by DirectSample arrives, it will be selected into the sample if it is a 1-bit (Line 6). Note that we do not need to check the element's hash value as it has been guaranteed to be in $R_{\ell}$ by DirectSample. After the element is processed (Line 6- 11), the function call at Line 12 gives the next stream location, at which the stream element will need to be checked. By using the direct sampling technique, our method intuitively is much faster than GT's method, which we will show later through both theoretical analysis and empirical study.

In the case where the sample becomes overflow after the insertion of a new element (Line 8), the sample level will be increased by one (Line 9). All the stream elements that are currently in the sample but whose hash values do not belong to $R_{\ell}$ will be discard from the sample (Line 11). The sample level will keep increasing until the sample is not overflowed (Line 8). There is a possibility that the sample level can exceed $\lfloor\log \mathrm{p}\rfloor$. If that happens, the algorithm fails, but we will later 


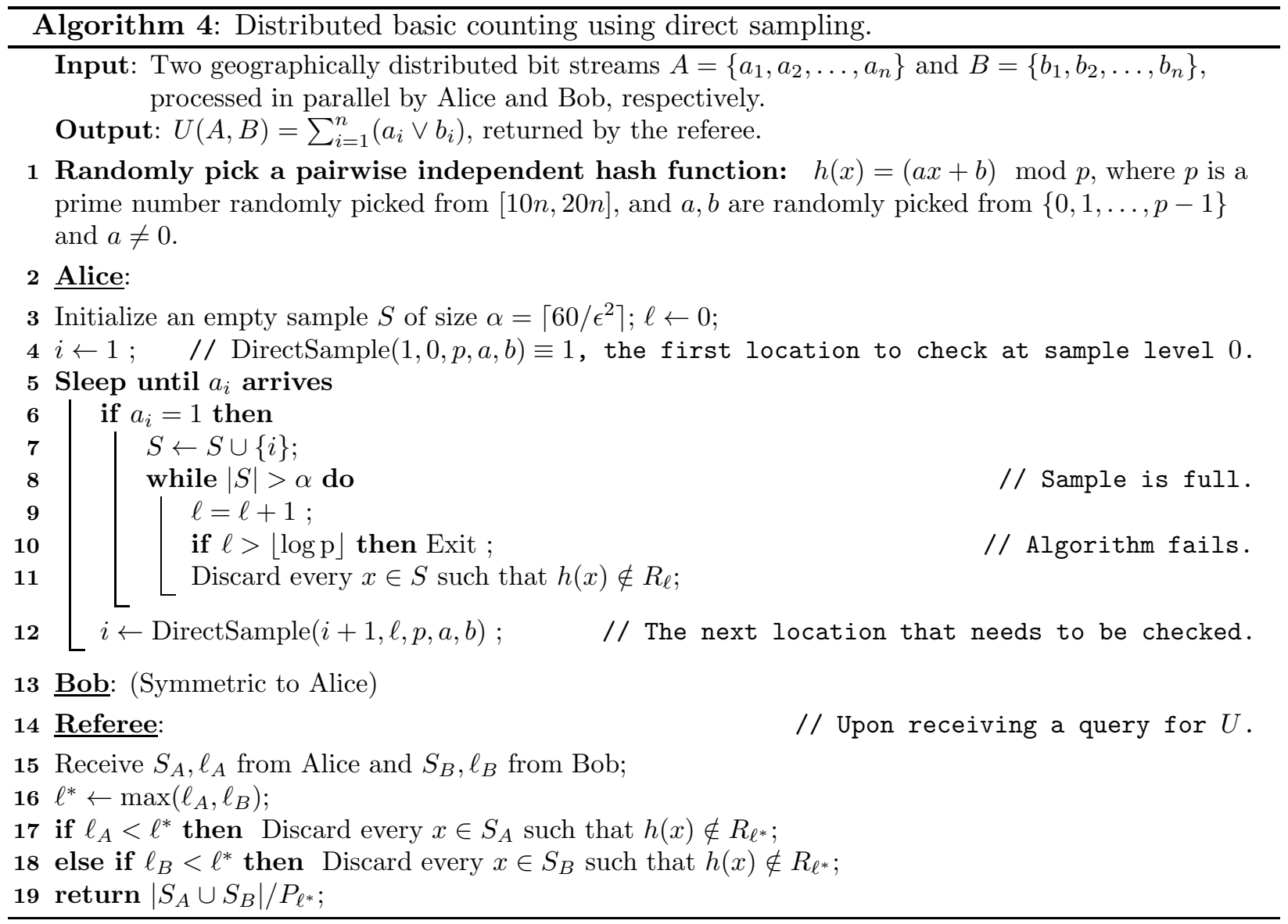

show the probability of this event is very low. The procedure at the referee to answer a query for $U$ (Line 15-19) is the same as the one in Algo. 1.

\subsection{Correctness}

Note that our method still uses the coordinated adaptive sampling on the high level, but uses DirectSample for speedup, so the correctness proof of our method follows a parallel structure of the proof by Gibbons and Tirthapura [14]. We present the entire proof here, because the hash function used in our method is different and also for completeness.

The only difference between Algo. 1 and 4 lies in the behavior of the stream processors. The processors in Algo. 1 process every incoming stream element, whereas the processors Algo. 4 ignore those stream elements that have no possibility to be selected into the sample. Due to the correctness of DirectSample used by Algo. 4 for faster sampling, given the same data stream and using the same hash function, a processor using Algo. 1 will end up with the same collection of selected elements as a processor using Algo. 4 after processing the stream. Recall that the behavior of the referee from both algorithms is identical. So, given the same distributed streams and the same hash function for coordinated sampling, the answer returned by the referee of Algo. 1 will be the same as the one returned by the referee of Algo. 4. Thus, we can prove the correctness of Algo. 4 by showing the correctness proof of Algo. 1. 
Let $1_{A}=\left\{1 \leq i \leq n \mid a_{i}=1\right\}, 1_{B}=\left\{1 \leq i \leq n \mid b_{i}=1\right\}$, and $1_{U}=\left\{1 \leq i \leq n \mid a_{i} \vee b_{i}=1\right\}$. Because the sampling procedures at both the processors and the referee are coordinated, the sample obtained by the referee at the query time can be viewed as the one whose elements are directly sampled by the referee from the stream $1_{U}$. The quality of the answer returned by the referee is solely determined by this sample, so our correctness proof will be focused on the analysis of this sample. Recall that $U(A, B)=\sum_{i=1}^{n}\left(a_{i} \vee b_{i}\right)$ and thus $\left|1_{U}\right|=U(A, B)$. We will use $U$ to represent $U(A, B)$ if the context is clear. The following process is hypothetical, visualized only to serve the correctness proof, and does not actually happen at the referee. The referee maintains $\lfloor\log \mathrm{p}\rfloor+1$ samples of level $\ell=0,1, \ldots,\lfloor\log \mathrm{p}\rfloor$. For each $i \in 1_{U}$, the element $i$ is selected into the sample of level $\ell$ if and only if $h(i) \in R_{\ell}$.

Definition 5.1. For $\ell=0,1, \ldots,\lfloor\log \mathrm{p}\rfloor$ and each $i \in 1_{U}$, let the indicator random variable

$$
X_{\ell, i}= \begin{cases}1, & \text { if } h(i) \in R_{\ell} \\ 0, & \text { otherwise }\end{cases}
$$

Definition 5.2. For $\ell=0,1, \ldots,\lfloor\log \mathrm{p}\rfloor$, let the random variable $X_{\ell}=\sum_{i \in 1_{U}} X_{\ell, i}$

Definition 5.3. For $\ell=0,1, \ldots,\lfloor\log \mathrm{p}\rfloor$, we say: (1) the sample of level $\ell$ is bad, if $\left|X_{\ell} / P_{\ell}-U\right|>$ $\epsilon U$. (2) random event $B_{\ell}$ happens if level $\ell$ is bad.

Fact 5.1. For a particular $\ell \in\{0,1, \ldots,\lfloor\log \mathrm{p}\rfloor\}$ and any $i, j \in 1_{U}$ and $i \neq j$, the random variables $X_{\ell, i}$ and $X_{\ell, j}$ are pairwise independent.

Fact 5.2. For any $\ell \in\{0,1, \ldots,\lfloor\log \mathrm{p}\rfloor\}$ and any $i \in 1_{U}: 1 / 2^{\ell+1} \leq \operatorname{Pr}\left[X_{\ell, i}=1\right]=P_{\ell} \leq 1 / 2^{\ell}$

Lemma 5.1. For each $\ell=0,1, \ldots,\lfloor\log \mathrm{p}\rfloor: \mathrm{E}\left[X_{\ell}\right]=U P_{\ell}$ and $U / 2^{\ell+1} \leq \mathrm{E}\left[X_{\ell}\right] \leq U / 2^{\ell}$

Proof. $\mathrm{E}\left[X_{\ell}\right]=\mathrm{E}\left[\sum_{i \in 1_{U}} X_{\ell, i}\right]=\sum_{i \in 1_{U}} \mathrm{E}\left[X_{\ell, i}\right]=\sum_{i \in 1_{U}} \operatorname{Pr}\left[X_{\ell, i}=1\right]=\sum_{i \in 1_{U}} P_{\ell}=U P_{\ell}$, where the second equality uses the linearity of expectation. Using Fact 5.2, the lemma is proved.

Lemma 5.2. For each $\ell=0,1, \ldots,\lfloor\log \mathrm{p}\rfloor: \operatorname{Var}\left[X_{\ell}\right] \leq \mathrm{E}\left[X_{\ell}\right]$

Proof.

$$
\begin{aligned}
\operatorname{Var}\left[X_{\ell}\right] & =\operatorname{Var}\left[\sum_{i \in 1_{U}} X_{\ell, i}\right] \\
& =\sum_{i \in 1_{U}} \operatorname{Var}\left[X_{\ell, i}\right] \quad(\text { Fact 5.1) } \\
& =\sum_{i \in 1_{U}}\left(\mathrm{E}\left[X_{\ell, i}^{2}\right]-\mathrm{E}^{2}\left[X_{\ell, i}\right]\right) \\
& =\sum_{i \in 1_{U}}\left(\operatorname{Pr}\left[X_{\ell, i}=1\right]-\left(\operatorname{Pr}\left[X_{\ell, i}=1\right]\right)^{2}\right) \\
& \leq \sum_{i \in 1_{U}} \operatorname{Pr}\left[X_{\ell, i}=1\right] \\
& =\mathrm{E}\left[X_{\ell}\right]
\end{aligned}
$$


Lemma 5.3. For each $\ell=0,1, \ldots,\lfloor\log \mathrm{p}\rfloor: \operatorname{Pr}\left[B_{\ell}\right] \leq 2^{\ell+1} /\left(\epsilon^{2} U\right)$

Proof.

$$
\begin{aligned}
\operatorname{Pr}\left[B_{\ell}\right] & =\operatorname{Pr}\left[\left|\frac{X_{\ell}}{P_{\ell}}-U\right|>\epsilon U\right]=\operatorname{Pr}\left[\left|X_{\ell}-U P_{\ell}\right|>\epsilon U P_{\ell}\right] \\
& =\operatorname{Pr}\left[\left|X_{\ell}-\mathrm{E}\left[X_{\ell}\right]\right|>\epsilon \mathrm{E}\left[X_{\ell}\right]\right] \quad \text { (Lemma 5.1) } \\
& <\frac{\operatorname{Var}\left[X_{\ell}\right]}{\left(\epsilon \mathrm{E}\left[X_{\ell}\right]\right)^{2}} \quad \text { (Chebyshev's Inequality) } \\
& \leq \frac{1}{\epsilon^{2} \mathrm{E}\left[X_{\ell}\right]} \quad \text { (Lemma 5.2) } \\
& =\frac{2^{\ell+1}}{\epsilon^{2} U} \quad \text { (Lemma 5.1) }
\end{aligned}
$$

Definition 5.4. Let $\omega$ be the lowest numbered level such that $\mathrm{E}\left[X_{\omega}\right]<\alpha / 2$.

If $U \leq \alpha$, Algo. 4 will certainly return the exact value of $U$, so we only consider the interesting case where $U>\alpha=\left\lceil 60 / \epsilon^{2}\right\rceil$.

Lemma 5.4. Level $\omega$ exists and $0<\omega<\lfloor\log \mathrm{p}\rfloor$.

Proof. (1) $\omega>0$ is because $\mathrm{E}\left[X_{0}\right]=U>\alpha$ but $\mathrm{E}\left[X_{\omega}\right]<\alpha / 2$. (2) We prove $\omega<\lfloor\log \mathrm{p}\rfloor$ by showing that $\mathrm{E}\left[X_{\lfloor\log \mathrm{p}\rfloor-1}\right]<\alpha / 2$. Note that $p$ is prime number from $[10 n, 20 n]$, so $\lfloor\log \mathrm{p}\rfloor-1>\log n$. It follows that

$$
\begin{aligned}
\mathrm{E}\left[X_{\lfloor\log \mathrm{p}\rfloor-1}\right] & \leq \frac{U}{2^{\lfloor\log \mathrm{p}\rfloor-1} \quad(\text { Lemma } 5.1)} \\
& \leq \frac{U}{2^{\log n}} \leq \frac{n}{2^{\log n}}=1<\frac{\alpha}{2}=\frac{\left\lceil 60 / \epsilon^{2}\right\rceil}{2}
\end{aligned}
$$

Lemma 5.5. $\sum_{\ell=0}^{\omega} \operatorname{Pr}\left[B_{\ell}\right]<\frac{4}{15}$.

Proof. By Definition 5.4, we have $\mathrm{E}\left[X_{\omega-1}\right] \geq \alpha / 2 \geq 30 / \epsilon^{2}$. Combining the fact that $\mathrm{E}\left[X_{\omega-1}\right] \leq$ $U / 2^{\omega-1}$ (Lemma 5.1), we have $U / 2^{\omega-1} \geq 30 / \epsilon^{2}$, i.e., $2^{\omega-1} /\left(\epsilon^{2} U\right) \leq 1 / 30$. It follows that,

$$
\begin{aligned}
\sum_{\ell=0}^{\omega} \operatorname{Pr}\left[B_{\ell}\right] & <\sum_{\ell=0}^{\omega} \frac{2^{\ell+1}}{\epsilon^{2} U} \quad \text { (Lemma 5.3) } \\
& =\frac{2}{\epsilon^{2} U}\left(2^{\omega+1}-1\right)<\frac{8 \cdot 2^{\omega-1}}{\epsilon^{2} U} \leq \frac{4}{15}
\end{aligned}
$$

Recall that $\ell^{*}$ is the sample level at which the referee answer the query (Line 14, Algo. 1 and Line 16 of 4$)$.

Definition 5.5. For $\ell=0,1, \ldots,\lfloor\log \mathrm{p}\rfloor+1$, we say the random event $S_{\ell}$ happens if $\ell=\ell^{*}$, i.e., the referee uses the sample level $\ell^{*}=\ell$ to answers the query. 
Lemma 5.6. $\operatorname{Pr}\left[S_{\omega+1} \vee \ldots \vee S_{\lfloor\log \mathrm{p}\rfloor+1}\right] \leq 1 / 30$

Proof. If the random event $S_{\omega+1} \vee \ldots \vee S_{\lfloor\log \mathrm{p}\rfloor+1}$ happens, it means the algorithm cannot use a level numbered smaller than $\omega+1$ to answer the query. It follows that $X_{\omega}>\alpha$. So,

$$
\begin{aligned}
\operatorname{Pr}\left[S_{\omega+1} \vee \ldots \vee S_{\lfloor\log \mathrm{p}\rfloor+1}\right] & \leq \operatorname{Pr}\left[X_{\omega}>\alpha\right]=\operatorname{Pr}\left[X_{\omega}-\mathrm{E}\left[X_{\omega}\right]>\alpha-\mathrm{E}\left[X_{\omega}\right]\right] \\
& \leq \operatorname{Pr}\left[X_{\omega}-\mathrm{E}\left[X_{\omega}\right]>\alpha-\frac{\alpha}{2}\right] \quad \text { (Definition 5.4) } \\
& \leq \frac{\operatorname{Var}\left[X_{\omega}\right]}{(\alpha / 2)^{2}} \quad \text { (Chebyshev's Inequality) } \\
& \leq \frac{\mathrm{E}\left[X_{\omega}\right]}{\alpha^{2} / 4} \quad \text { (Lemma 5.2) } \\
& \leq \frac{2}{\alpha} \quad(\text { Lemma 5.4) } \\
& =\frac{\epsilon^{2}}{30} \leq \frac{1}{30}
\end{aligned}
$$

Lemma 5.7. One instance of Algo. 4 returns an $\epsilon$-estimate of $U$ with a constant probability of at least $3 / 10$.

Proof. The algorithm can fail for two possibilities: (1) it stops at a sample level $\ell \leq\lfloor\log \mathrm{p}\rfloor$, but the level is bad; or (2) it reaches the sample level $\lfloor\log \mathrm{p}\rfloor+1$ and the algorithm just exits (Line 10, Algo. 4). So,

$$
\begin{aligned}
\operatorname{Pr}[\text { failure }] & =\operatorname{Pr}\left[\left(S_{0} \wedge B_{0}\right) \vee\left(S_{1} \wedge B_{1}\right) \vee \ldots \vee\left(S_{\lfloor\log \mathrm{p}\rfloor} \wedge B_{\lfloor\log \mathrm{p}\rfloor}\right) \vee S_{\lfloor\log \mathrm{p}\rfloor+1}\right] \\
& \leq \operatorname{Pr}\left[B_{0} \vee \ldots \vee B_{\omega}\right]+\operatorname{Pr}\left[S_{\omega+1} \vee \ldots \vee S_{\lfloor\log \mathrm{p}\rfloor+1}\right] \\
& \leq \sum_{\ell=0}^{\omega} \operatorname{Pr}\left[B_{\ell}\right]+\operatorname{Pr}\left[S_{\omega+1} \vee \ldots \vee S_{\lfloor\log \mathrm{p}\rfloor+1}\right] \\
& \leq \frac{4}{15}+\frac{1}{30} \quad(\text { Lemma } 5.5 \text { and } 5.6) \\
& =\frac{3}{10}
\end{aligned}
$$

Let $\beta=\lceil 24 \ln (1 / \delta)\rceil$. The next theorem shows that we can further reduce the failure probability of Algo. 4 to the user input parameter $\delta$ by running $\beta$ independent instances of Algo. 4 and returning the median of the answers of those $\beta$ instances as the estimate of $U$. 
Theorem 5.1 (Correctness of Algo. 4). The median of the answers returned by $\beta=\lceil 24 \ln (1 / \delta)\rceil$ independent instances (using different hash functions picked randomly and independently) of Algo. 4 is an $(\epsilon, \delta)$-estimate of $U$.

Proof. For $i=1,2, \ldots, \beta$, let the indicator random variable $Y_{i}=1$ if the $i$ th instance does not return an $\epsilon$-estimate; $Y_{i}=0$, otherwise. Due to Lemma 5.7, we already have $\operatorname{Pr}\left[Y_{i}=1\right] \leq 3 / 10$. Let binomial random variable $Y=\sum_{i=1}^{\beta} Y_{i}$, then

$$
\mathrm{E}[Y]=\mathrm{E}\left[\sum_{i=1}^{\beta} Y_{i}\right]=\sum_{i=1}^{\beta} \mathrm{E}\left[Y_{i}\right]=\sum_{i=1}^{\beta} \operatorname{Pr}\left[Y_{i}=1\right] \leq \frac{3}{10} \beta
$$

If the median of the $\beta$ independent answers is not an $\epsilon$-estimate, it means that more than $\beta / 2$ answers are not $\epsilon$-estimate, i.e., $Y>\beta / 2$. We want to bound $\operatorname{Pr}[Y>\beta / 2]$.

For proof purpose, we define another binomial random variable $X=\sum_{i=1}^{\beta} X_{i}$, where each $\operatorname{Pr}\left[X_{i}=1\right]=3 / 10$, and thus $\mathrm{E}[X]=3 \beta / 10$.

$$
\begin{aligned}
\operatorname{Pr}\left[X>\frac{\beta}{2}\right] & =\operatorname{Pr}\left[X>\left(1+\frac{2}{3}\right) \mathrm{E}[X]\right] \leq \exp \left(\frac{-\mathrm{E}[X](2 / 3)^{2}}{3}\right) \quad \text { (Chernoff Bound) } \\
& \leq \exp \left(-\frac{3}{10} 24\left(\ln \frac{1}{\delta}\right) \frac{4}{27}\right) \leq \delta^{48 / 45} \leq \delta
\end{aligned}
$$

Note that $X$ and $Y$ are both binomial random variables of the form $X=B\left(\beta, p_{1}\right)$ and $Y=$ $B\left(\beta, p_{2}\right)$, but $p_{1}=3 / 10 \geq p_{2}$, so it is obvious:

$$
\operatorname{Pr}\left[Y>\frac{\beta}{2}\right] \leq \operatorname{Pr}\left[X>\frac{\beta}{2}\right] \leq \delta
$$

That is, the probability that median of the answers returned by the $\beta$ independent instances of Algo. 4 is not an $\epsilon$-estimate is no more than $\delta$.

\subsection{Time complexity}

Theorem 5.2 (Worst-case total time cost). Algo. 4 spends $\Theta(n \log (1 / \delta))$ time in the worst case for processing a bit stream of size $n$ for maintaining an $(\epsilon, \delta)$-estimate of $U$.

Proof. The total time cost of Algo. 4 for processing a stream includes the time cost for (1) the DirectSample function calls (Line 12), (2) inserting the selected 1-bits into the sample at various levels (Line 7), and (3) increasing the sample level when the sample is full (Line 8-11).

Suppose $d_{1}, d_{2}, \ldots, d_{t}$, for some $t \geq 1$, is the sequence of integers returned by the DirectSample function calls during the stream processing. Note that:

$$
t+\sum_{i=1}^{t} d_{i} \geq n \quad \text { and } \quad t+\sum_{i=1}^{t-1} d_{i}<n
$$

The total processing time is bounded by

$$
\sum_{i=1}^{t} O\left(\log d_{t}\right)+O(t)+O(\alpha\lfloor\log \mathrm{p}\rfloor)
$$


where the three terms capture the time cost of type (1), (2), and (3), respectively. Following Equation 6,

$$
\begin{aligned}
& \sum_{i=1}^{t} O\left(\log d_{i}\right)+O(t)+O(\alpha\lfloor\log \mathrm{p}\rfloor) \\
= & \sum_{i=1}^{t-1} O\left(\log d_{i}\right)+O\left(\log d_{t}\right)+O(t)+O\left(\left\lceil 60 / \epsilon^{2}\right\rceil\lfloor\log \mathrm{p}\rfloor\right) \\
= & \sum_{i=1}^{t-1} O\left(d_{i}\right)+O(\log n)+O(n)+O\left(1 /\left(\epsilon^{2}\right) \log n\right) \\
= & \left.O(n)+O(n)+O\left(1 /\left(\epsilon^{2}\right) \log n\right) \quad \text { (Inequality } 5\right) \\
= & O(n)
\end{aligned}
$$

The above bound is also tight. For example, when the stream has all 0-bits, the sample will always be empty and thus will always be at level 0, meaning every stream element will be checked, giving a total time cost of at least $\Theta(n)$. Therefore, the worst case total time cost of one instance of Algo. 4 is $\Theta(n)$. The claim in the theorem follows due to the fact that we need to run $\beta$ instances of Algo. 4 (Theorem 5.1) in order to maintain an $(\epsilon, \delta)$-estimate of $U$.

Comment: (1) $\Theta(n \log (1 / \delta))$ is GT's total time cost in any case, but it rarely occurs with our method. (2) It is necessary to spend $\Theta(n \log (1 / \delta))$ in the worst case, because in the case all stream elements are 0 -bits, we need to check every stream element if we want to estimate $U$ with a relative error guarantee.

Theorem 5.3 (Worst-case per-element time cost). Algo. 4 spends $\Theta(\log (1 / \delta) \log n)$ time in the worst case for processing one stream element in order to maintain an $(\epsilon, \delta)$-estimate of $U$.

Proof. The time cost of Algo. 4 for processing one stream element in the worst case includes those for (1) the function call for DirectSample (Line 12), (2) inserting the element into the sample if it is a 1-bit (Line 7), and (3) increasing the sample level when the sample is full (Line 8-11).

The time cost of type (1) is bounded by $O(\log n)$. The time cost of type (2) is bounded by $O(1)$. Next, let's look at the time cost of type (3). We organize all the elements in the sample into multiple linked lists. Each linked list is the collection of elements who share the same highest sample level through which the element will still remain in the sample. We call this level as the list's surviving level. Note that there can be at most $\lfloor\log \mathrm{p}\rfloor+1$ linked lists, because the algorithm maintains no more than $\lfloor\log \mathrm{p}\rfloor+1$ sample levels. Every time we need to insert a new element into the sample, there will be two cases.

Case 1: the sample is not full. We will first find which linked list the new element belongs to. This takes $O(\lfloor\log \mathrm{p}\rfloor)=O(\log n)$ time. We will then insert the new element into that linked list which takes $O(1)$ time. So altogether, the time cost of type $(3)$ is $O(\log n)$.

Case 2: if the sample is full, we will discard the elements from the sample in a lazy manner. We will first keep increasing the sample level until we get at least one linked list $L$ whose surviving level is smaller than the current sample level. This takes no more than $O(\lfloor\log \mathrm{p}\rfloor)=O(\log n)$ time, because the algorithm maintains no more than $\lfloor\log \mathrm{p}\rfloor+1$ levels. We will then delete an element from $L$. Then we insert the new element into the appropriate linked list that the new element 
belongs to. This again takes no more than $O(\lfloor\log \mathrm{p}\rfloor)=O(\log n)$ time, since we need to find that right linked list among at most $\lfloor\log \mathrm{p}\rfloor+1$ linked lists. So altogether, the time cost of type (3) in this case is $O(\log n)$. This bound is also tight. For example, suppose at some certain point of time there are $\Theta(\log n)$ linked list being maintained in the sample. In that case, finding the appropriate linked list to insert the new element will take $\Theta(\log n)$ time in the worst case, meaning the time cost of type (3) will be indeed $\Theta(\log n)$.

Add the time cost of type (1), (2), and (3), we get the worst-case time cost for processing one stream element by one instance of Algo. 4 is $\Theta(\log n)$. The claim in the theorem then follows due to the fact that we need to run $\beta$ instances of Algo. 4 (Theorem 5.1) in order to maintain an $(\epsilon, \delta)$-estimate of $U$.

Comment: In practice, when the stream size is large, only a very small portion of the stream will be checked in our method. This indicates the probability of any particular element being processed by more than a constant number of instances of Algo. 4 is very low. So, our method's worst-case time cost per element will be $O(\log n)$ with high probability.

Intuitively, the new ingredient DirectSample can significantly speed up the stream processing in practice. Next, we show that the average total time cost of Algo. 4 for processing a $\gamma$-random bit stream is indeed sublinear of the stream size. Recall that the notion of $\gamma$-random bit stream (Definition 1.2) can fit into the distribution of many real-world streams.

Theorem 5.4 (Average-case total time cost for $\gamma$-random bit stream). The average time cost of Algo. 4 for processing a $\gamma$-random bit stream of size $n$ is $O\left(\frac{1}{\gamma \epsilon^{2}} \log ^{2} n \log \frac{1}{\delta}\right)$ in the maintenance of an $(\epsilon, \delta)$-estimate for $U$.

Proof. There are no more than $\lfloor\log \mathrm{p}\rfloor$ sample levels and the sample size is $\alpha=\left\lceil 60 / \epsilon^{2}\right\rceil$, so the number of 1-bits that are selected by one instance of Algo. 4 over the course of the stream processing is no more than $\alpha\lfloor\log \mathrm{p}\rfloor$, which is also true even if the sample level exceeds $\lfloor\log \mathrm{p}\rfloor$ as the algorithm will exit in that case (Line 10).

On the other hand, in a $\gamma$-random bit stream, the probability that a stream location selected by DirectSample has a 1-bit is at least $\gamma$. So, for each 1-bit that is selected over the course of stream processing, we have in average $1 / \gamma^{2}$ before DirectSample instances of DirectSample function calls before DirectSample actually returns a stream location which indeed contains a 1-bit. Therefore, in average, there are no more than $(1 / \gamma) \alpha\lfloor\log \mathrm{p}\rfloor$ stream elements that have been checked by Algo. 4. By Theorem 5.3, we know the worst-case per-element time cost by one instance of Algo. 4 is $\Theta(\log n)$, we get the average-case total time cost by one instance of Algo. 4 for processing a $\gamma$-random bit stream of size $n$ is $O(\log n) \cdot(1 / \gamma) \alpha\lfloor\log \mathrm{p}\rfloor=O\left(\left(1 /\left(\gamma \epsilon^{2}\right)\right) \log ^{2} n\right)$.

The claim in the theorem then follows from the fact that we need to run $\beta$ instances of Algo. 4 (Theorem 5.1) in order to guarantee an $(\epsilon, \delta)$-estimate guarantee of $U$.

Comment: (1) Our method is significantly faster than GT's in practice, providing the first sublinear-time algorithm in the average case for the distributed basic counting problem over most real-world streams. (2) Our method provides the users with the new tradeoff that a lower accuracy demand (a larger value for $\epsilon$ ) promises a faster processing speed, whereas GT's is $\Theta(n \log (1 / \delta))$ in any case.

\footnotetext{
${ }^{2}$ The expectation of a geometric random variable with parameter $\gamma$.
} 


\subsection{Space and communication complexity}

Theorem 5.5 (Space and communication cost). To maintain an $(\epsilon, \delta)$-estimate of $U$ : (1) the workspace at each processor is $O\left(\left(1 / \epsilon^{2}+\log n\right) \log (1 / \delta) \log n\right)$ bits. (2) the memory usage by the referee is $O\left(\left(1 / \epsilon^{2}\right) \log (1 / \delta) \log n\right)$ bits. (3) The communication cost per query between each processor and the referee is $O\left(\left(1 / \epsilon^{2}\right) \log (1 / \delta) \log n\right)$ bits.

Proof. The workspace at processors. The workspace by each processor is $O\left(\left(1 / \epsilon^{2}+\log n\right) \log (1 / \delta) \log n\right)$ bits, including the following memory usages.

- The memory space for storing the sample system. The sample maintained by each processor contains no more than $\alpha=\left\lceil 60 / \epsilon^{2}\right\rceil$ integers from the range $[1, n]$, using no more than $O(\alpha \log n)=O\left(\left(1 / \epsilon^{2}\right) \log n\right)$ bits. The memory usage for recording the hashing function is $O(\log n)$ bits, because the three parameters $a, b, p$ that define the hash function $h(x)=(a x+b) \bmod p$ are all bounded $O(n)$ bits (Section 3.2). The number of bits used for recording the current sample level is bounded by $O(\log \log p)=O(\log \log n)$, because the sample level is no more than $\lfloor\log \mathrm{p}\rfloor$. There are $\beta=\lceil 24 \ln (1 / \delta)\rceil$ instances running in parallel on each processor, so the total memory cost for the sample system at each processor is $\left.O\left(\left(1 / \epsilon^{2}\right) \log n+\log n+\log n \log n\right) \log (1 / \delta)\right)=O\left(\left(1 / \epsilon^{2}\right) \log (1 / \delta) \log n\right)$ bits.

- The stack memory cost for the DirectSample function call (Line 12, Algo. 4). It has been proved in Corollary 4.1 that the space usage by one DirectSample function call is no more than $O(\log n \cdot \min (\log a, \log d))=O\left(\log ^{2} n\right)$ bits. There are $\beta=\lceil 24 \ln (1 / \delta)\rceil$ instances running in parallel on each processor, so the total memory cost for the DirectSample function calls is no more than $O\left(\log (1 / \delta) \log ^{2} n\right)$ bits.

The workspace at the referee. The memory needs by the referee is only for storing the samples it has received from the two processors, where the space usage of the samples from each processor is $O\left(\left(1 / \epsilon^{2}\right) \log (1 / \delta) \log n\right)$ bits.

The communication cost per query and per link. Upon the arrival of a query at the referee, the only information that the referee needs to collect from the processors is the samples maintained by the processors. So, the communication cost per query and per link is $O\left(\left(1 / \epsilon^{2}\right) \log (1 / \delta) \log n\right)$ bits, the space usage of the samples sent from one processor.

Comment: Compared with the space usage of GT's, each processor's extra space cost in our method is $O\left(\log ^{2} n \log (1 / \delta)\right)$ bits due to the DirectSample function calls. This extra space usage is a lower order term compared with GT's space usage when the value of $\epsilon$ is reasonably small (for ex., $\epsilon \leq 0.1)$. Further, this extra space cost occurs only $O(\log n)$ times for each algorithm instance in average during the stream processing, because there are only $O(\log n)$ DirectSample function calls within one algorithm instance in average over the course of stream processing. In practice the stream size $n$ is often very large, which is usually the case in stream processing (otherwise there is no need to design space-efficient streaming algorithms), the stream locations that are returned by DirectSample within one algorithm instance will be very sparse, meaning the probability that those $\beta$ parallel instances will call the DirectSample function at the same time is very low. So, in practice, the extra space used for stack maintenance in the $\beta$ instances will be only $O\left(\log ^{2} n\right)$ bits and occurs only $O(\log n)$ times with high probability. We will later show through experiments with both real-world and synthetic data that this extra space usage is too negligible to be detected by the OS. 


\subsection{Extension: multiple streams and multiple processors}

It is trivial to extend our method for maintaining an $(\epsilon, \delta)$-estimate for the distributed basic counting over $k>2$ streams that are processed by $k$ processors, for some constant $k$. The procedure at each of these $k$ processors will be exactly the same as the procedure in the 2 -stream scenario. The difference is when a query arrives, the referee needs to collect the samples and their sample levels from all the processors, and then run the following procedure to generate the estimate for $U$ (Equation 1). The correctness proof and time and space complexity analysis for the 2-stream case can be directly applied to this $k$-stream scenario.

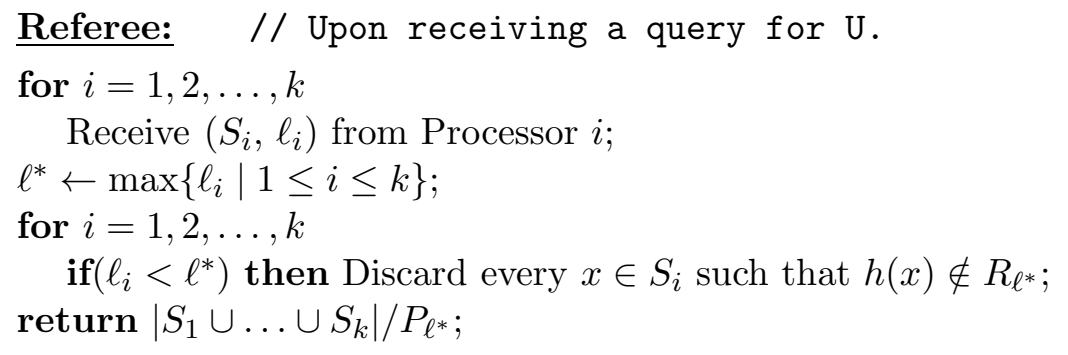

\section{Experiments}

Main message. Note that our method does not change the accuracy of GT's coordinated adaptive sampling technique (a.k.a. distinct sampling), whose accuracy in estimating the basic counting has been well studied and validated by prior work $[13,10]^{3}$. Thus, in this section, we only want to demonstrate the time and space efficiency of our method compared with GT's through experiments with both real-world and synthetic data. The main messages from the experimental study are: (1) Our method is exponentially faster than GT's method, whose processing time is linear of the stream size. (2) Our method's processing speed increases when the accuracy demand decreases (the value of $\epsilon$ increases), while GT's method's processing speed does not change regardless of the value of $\epsilon$. (3) Our method does not introduce any detectable space overhead, regardless of the data sets and the value of $\epsilon$, compared with the space cost by GT's. All the above observations are perfectly consistent with the theoretical results summarized in Table 1.1.

System setup and data source. All of our experiments were conducted on a Dell Precision T5500s machine that has a $2.13 \mathrm{GHz}$ Quad-core E5506 CPU with $4 \mathrm{M}$ cache, but no parallelism was used. The machine runs 64-bit Ubuntu 10.04 Desktop and has 8GB DDR3 ECC 1066MHz SDRAM. We faithfully implemented the coordinated adaptive sampling both with and without using the DirectSample technique (Algo. 1 and 4) using the C++ programming language ${ }^{4}$. All executables were built by GCC 4.4.3. We used the following real-world and synthetic bit sequences in our experiments:

- Audio Bible ${ }^{5}$. We concatenated all the MP3 files of the audio Bible. It has a total of $919,658,056$ bits, of which 460, 805, 446 are 1-bits.

\footnotetext{
${ }^{3}$ In particularly, the hash function used in [10] is the same as the one we use in this paper.

${ }^{4}$ The C++ source code can be downloaded at: http://penguin.ewu.edu/ bojianxu/publications.

${ }^{5}$ http://spiritlessons.com/Documents/Bible/NIV_MP3_Bible/NIV_MP3_Bible.zip
} 


\begin{tabular}{|c|c|c|c|c|c|c|c|}
\hline Data & Methods & $\epsilon=\mathbf{0 . 0 1}$ & $\epsilon=0.02$ & $\epsilon=\mathbf{0 . 0 5}$ & $\epsilon=\mathbf{0 . 1}$ & $\epsilon=\mathbf{0 . 2}$ & $\epsilon=\mathbf{0 . 5}$ \\
\hline \multirow{3}{*}{$\begin{array}{l}\text { Audio Bible } \\
460,805,446 \text { 1-bits }\end{array}$} & GT's & 18.7823 & 18.3439 & 18.2506 & 18.2531 & 18.2386 & 18.2312 \\
\hline & Ours & 3.6273 & 1.0784 & 0.2841 & 0.0528 & 0.0285 & 0.0046 \\
\hline & Speedup & $>5 x$ & $>17 x$ & $>64 x$ & $>345 x$ & $>639 \mathrm{x}$ & $>3952 \mathrm{x}$ \\
\hline \multirow{3}{*}{$\begin{array}{l}\text { Video of President } \\
710,447,850 \text { 1-bits }\end{array}$} & GT's & 28.8833 & 28.6107 & 28.4632 & 28.2885 & 28.4486 & 28.5082 \\
\hline & Ours & 3.2259 & 1.0117 & 0.3180 & 0.1055 & 0.0278 & 0.0076 \\
\hline & Speedup & $>8 x$ & $>28 x$ & $>89 x$ & $>268 x$ & $>1020 x$ & $>3727 x$ \\
\hline \multirow{3}{*}{$\begin{array}{l}\text { Earth Image } \\
789,808,848 \text { 1-bits }\end{array}$} & GT's & 31.9561 & 31.6179 & 31.5310 & 31.4197 & 31.5040 & 31.5009 \\
\hline & Ours & 3.1705 & 1.3294 & 0.2932 & 0.0843 & 0.0245 & 0.0066 \\
\hline & Speedup & $>10 x$ & $>23 x$ & $>107 x$ & $>372 x$ & $>1285 x$ & $>4725 \mathrm{x}$ \\
\hline \multirow{3}{*}{$\begin{array}{l}\text { Worldcup } 98 \\
257,380,419 \text { 1-bits }\end{array}$} & GT's & 12.9562 & 12.5322 & 12.5236 & 12.5224 & 12.5106 & 12.4987 \\
\hline & Ours & 5.4275 & 1.6266 & 0.2791 & 0.0678 & 0.0372 & 0.0070 \\
\hline & Speedup & $>2 x$ & $>7 x$ & $>44 \mathrm{x}$ & $>184 \mathrm{x}$ & $>336 x$ & $>1762 \mathrm{x}$ \\
\hline \multirow{3}{*}{$\begin{array}{l}\text { Synthetic-0.3 } \\
300,023,303 \text { 1-bits }\end{array}$} & GT's & 15.6868 & 15.2895 & 15.2082 & 15.1802 & 15.1939 & 15.1601 \\
\hline & Ours & 2.9945 & 1.4045 & 0.4531 & 0.0998 & 0.0531 & 0.0078 \\
\hline & Speedup & $>5 x$ & $>10 \mathrm{x}$ & $>33 x$ & $>152 \mathrm{x}$ & $>286 x$ & $>1923 x$ \\
\hline \multirow{3}{*}{$\begin{array}{l}\text { Synthetic-0.4 } \\
400,002,206 \text { 1-bits }\end{array}$} & GT's & 18.5577 & 17.5786 & 18.0129 & 17.9844 & 17.9933 & 17.9982 \\
\hline & Ours & 2.3465 & 0.8511 & 0.2100 & 0.0782 & 0.0306 & 0.0063 \\
\hline & Speedup & $>7 x$ & $>20 x$ & $>85 \mathrm{x}$ & $>229 x$ & $>586 x$ & $>2851 x$ \\
\hline
\end{tabular}

Table 2: The time cost (in seconds) of both GT's and our methods in the processing of multiple bit streams with different accuracy demands $\epsilon$. The processing speed of GT's does not change with $\epsilon$, while ours becomes faster when $\epsilon$ increases. In all cases, our method is significantly faster than GT's method.

- Video of President G. W. Bush's speech ${ }^{6}$. It has a total of $1,434,146,160$ bits, of which $710,447,850$ are 1-bits.

- NASA Earth image ${ }^{7}$. It has a total of 1, 575,903, 872 bits, of which 789, 808, 848 are 1-bits.

- Day 37 of Worldcup 98's network traffic ${ }^{8}$. It has a total of $893,788,160$ bits, of which 257,380, 419 are 1-bits.

- Two synthetic bit sequences, where the probabilities of having a 1-bit are 0.3 and 0.4, respectively. Each sequence has a total of 1,000,000,000 bits, of which 300,023,303 and 400, 002, 206 are 1-bits, respectively.

\subsection{Time efficiency}

Table 2 shows the total time cost of both methods in processing different data sets with different accuracy demands.

\footnotetext{
${ }^{6}$ https://ia600306.us.archive.org/23/items/Political_videos-GeorgeWBush20030904_5_224/Political_ videos-GeorgeWBush20030904_5_224.ogv

${ }^{7}$ http://eoimages.gsfc.nasa.gov/images/imagerecords/73000/73909/world. topo.bathy . 200412. $3 \times 21600 \times 10800$.png

${ }^{8}$ http://ita.ee.lbl.gov/html/contrib/WorldCup.html
} 


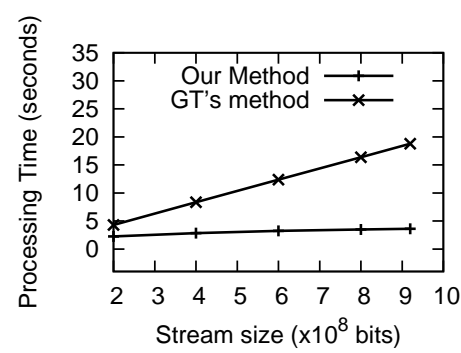

(a) Audio Bible

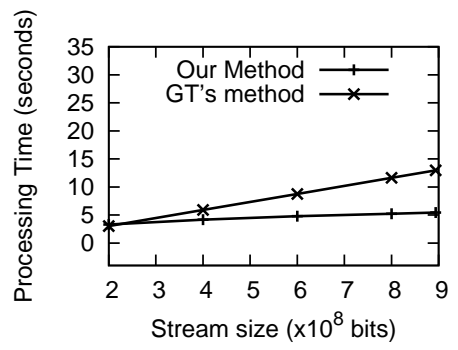

(d) Worldcup 98

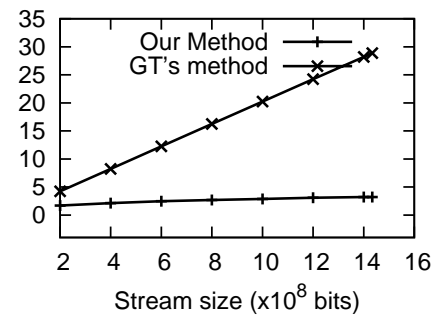

(b) Video of President

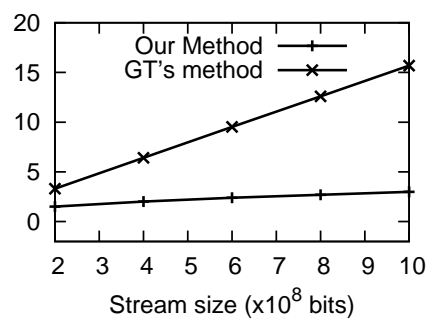

(e) Synthetic-0.3

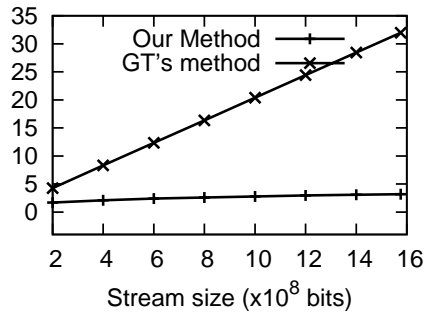

(c) Earth Image

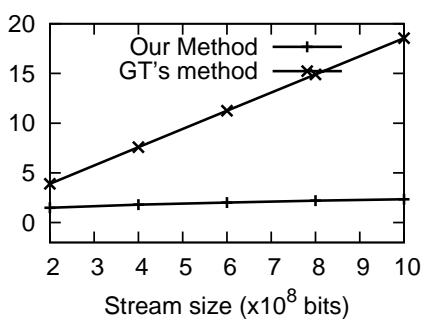

(f) Synthetic-0.4

Figure 3: Stream size vs. time, $\epsilon=0.01$. The processing time of GT's method is linear of the stream size, while ours is sublinear. Our method overall is much faster than GT's, especially when the stream size (the number of 1-bits in the stream, indeed) becomes larger.

Overall boosting. Our method overall is significantly faster than GT's by a factor of several to several thousand times. This speedup becomes more significant when the value of $\epsilon$ increases and/or the stream size (more precisely, the number of 1-bits in the stream) increases. When deployed in the real world, where the stream size is nearly unbounded, our method can save a a vast majority of the processing time and energy that is needed by GT's.

A new tradeoff. The time cost of GT's method is independent from the value of $\epsilon$, because their technique processes every stream element. However, our method becomes much faster when the value of $\epsilon$ increases. This is because a larger $\epsilon$ yields a smaller sample size, which makes the sample level be increased more often. Recall that a higher sample level selects stream elements with smaller probability, so it helps our DirectSample technique be able to skip more stream elements. This new feature in our method is important and useful, because it provides the user with a new trade-off that a lower accuracy demand will not only save memory space but also will speed up the data processing.

Even faster for longer streams. The more 1-bits are present in the stream, the more significant improvement our method makes against GT's method. This is because more 1-bits leads to a higher sample level during the stream processing, which yields a lower sampling probability. That helps DirectSample skip over more stream elements, leading to an overall faster processing speed.

Sublinear-linear time cost. Figure 3 shows that the time cost of GT's method is linear of the stream size, simply because their method processes every stream element, whereas our method's processing time is sublinear of the stream size. The figure again shows our method overall is much faster than GT's, especially when the stream size (the number of 1-bits in the stream, indeed) becomes larger for the reason that we have given above. The plot in Figure 3 shows the case 


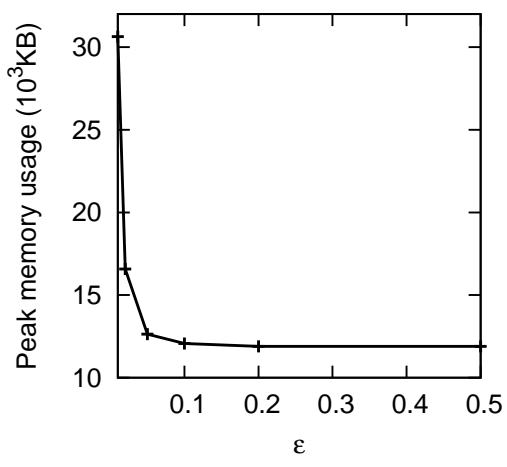

\begin{tabular}{|l|r|r|r|r|r|r|}
\hline Methods & $\epsilon=\mathbf{0 . 0 1}$ & $\epsilon=\mathbf{0 . 0 2}$ & $\epsilon=\mathbf{0 . 0 5}$ & $\epsilon=\mathbf{0 . 1}$ & $\epsilon=\mathbf{0 . 2}$ & $\epsilon=\mathbf{0 . 5}$ \\
\hline \hline GT's & 30,640 & 16,576 & 12,640 & 12,072 & 11,892 & 11,892 \\
\hline Ours & 30,640 & 16,576 & 12,640 & 12,072 & 11,892 & 11,892 \\
\hline Overhead & $\mathbf{0} \%$ & $\mathbf{0} \%$ & $\mathbf{0} \%$ & $\mathbf{0} \%$ & $\mathbf{0} \%$ & $\mathbf{0} \%$ \\
\hline
\end{tabular}

Figure 4: The space usage (in KB) of both methods in the processing of multiple bit streams with different $\epsilon$. The space usage of both methods increases when $\epsilon$ decreases and is independent from the stream size. There is no detectable extra space cost by our method compared with GT's.

where $\epsilon=0.01$. Figures regarding other $\epsilon$ values are given in the appendix, from which the same observations can be made.

\subsection{Space efficiency}

We measure the space usage of our programs by VmPeak minus the memory cost for storing the data sets. VmPeak is an entry in the /proc/ pid $\rangle /$ status file, provided by the Linux system. It captures the peak usage in KB of the total amount of virtual memory used by the process, including the memory cost for the code, data, and shared libraries plus the pages that have been swapped out. VmPeak represents the peak of the actual total memory cost of the process. Figure 6.2 shows the space usage of both methods.

Space usage only depends on $\epsilon$. The space usages of both methods are independent of stream size, but heavily depend upon the value of $\epsilon$. When the value of $\epsilon$ decreases, the space usage increases. This is consistent with the theoretical results - the space usage of one instance of GT's and our method are $O\left(\left(1 / \epsilon^{2}\right) \log n\right)$ bits and $O\left(\left(1 / \epsilon^{2}+\log n\right) \log n\right)$ bits, respectively. Note that we use the 64-bit unsigned long int to represent the stream size, so the impact of the $\log n$ term in the big-oh bounds becomes fixed.

No detectable extra space usage. For all data sets and all $\epsilon$ values, there is no detectable extra space usage by our method. That is, the extra space cost of $O\left(\log ^{2} n\right)$ bits by our method from the theoretical analysis is too negligible to be detected by the OS in practice.

\section{Conclusion}

In this paper, we designed DirectSample, a new technique for fast sampling, and used it in the coordinated adaptive sampling for distributed basic counting. Both the theoretical analysis and the experimental results show that our method is exponentially faster than the state-of-the-art GT's method. Further, the extra space usage by our method from the theoretical analysis is not only negligible in theory but also undetectable in practice. Our new method can potentially save a vast majority of processing time and energy needed by GT's method in the processing of real-world streams, whose size is nearly unbounded. We also believe the new DirectSample technique can be of other independent interest. 


\section{Acknowledgement}

The author acknowledges Atalay Mert İleri from Bilkent University for the helpful discussion on the proof of Lemma 4.1.

\section{References}

[1] N. Alon, P. B. Gibbons, Y. Matias, and M. Szegedy. Tracking algorithms for join and self-join sizes. In Proc. 18th ACM Symp. on Principles of Database Systems, pages 1-11, May 1999. Full version to appear in JCSS special issue for PODS'99.

[2] N. Alon, Y. Matias, and M. Szegedy. The space complexity of approximating the frequency moments. Journal of Computer and System Sciences, 58(1):137-147, 1999.

[3] B. Babcock, S. Babu, M. Datar, R. Motwani, and J. Widom. Models and issues in data stream systems. In Proc. 21st ACM Symp. on Principles of Database Systems (PODS), pages 1-16, 2002 .

[4] Z. Bar-Yossef, T. Jayram, R. Kumar, D. Sivakumar, and L. Trevisan. Counting distinct elements in a data stream. In Proc. 6th International Workshop on Randomization and Approximation Techniques (RANDOM), pages 1-10, 2002. Lecture Notes in Computer Science, vol. 2483, Springer.

[5] C. Busch and S. Tirthapura. A deterministic algorithm for summarizing asynchronous streams over a sliding window. In Proc. 24th Annual Symposium on Theoretical Aspects of Computer Science (STACS), pages 465-476, 2007.

[6] J.L. Carter and M.L. Wegman. Universal classes of hash functions. Journal of Computer and System Sciences, 18(2):143-154, 1979.

[7] G. Cormode, M. Garofalakis, S. Muthukrishnan, and R. Rastogi. Holistic aggregates in a networked world: Distributed tracking of approximate quantiles. In ACM SIGMOD International Conference on Management of Data (SIGMOD), pages 25-36, 2005.

[8] G. Cormode, S. Muthukrishnan, and K. Yi. Algorithms for distributed, functional monitoring. In ACM-SIAM Symposium on Discrete Algorithms (SODA), 2008.

[9] G. Cormode, S. Muthukrishnan, and W. Zhuang. What's different: Distributed, continuous monitoring of duplicate-resilient aggregates on data streams. In International Conference on Data Engineering (ICDE), pages 20-31, 2006.

[10] G. Cormode, S. Tirthapura, and B. Xu. Time-decaying sketches for robust aggregation of sensor data. SIAM Journal on Computing, 39(4):1309-1339, 2009. (Also in PODC2007).

[11] M. Datar, A. Gionis, P. Indyk, and R. Motwani. Maintaining stream statistics over sliding windows. SIAM Journal on Computing, 31(6):1794-1813, 2002.

[12] E. Fischer. The art of uninformed decisions: A primer to property testing. Science, 75:97-126, 2001. 
[13] P. Gibbons. Distinct sampling for highly-accurate answers to distinct values queries and event reports. In Proc. 27th International Conf. on Very Large Data Bases (VLDB), pages 541-550, 2001.

[14] P. Gibbons and S. Tirthapura. Estimating simple functions on the union of data streams. In Proc. ACM Symp. on Parallel Algorithms and Architectures (SPAA), pages 281-291, 2001.

[15] P. Gibbons and S. Tirthapura. Distributed streams algorithms for sliding windows. In Proc. ACM Symposium on Parallel Algorithms and Architectures (SPAA), pages 63-72, 2002.

[16] A. C. Gilbert, Y. Kotidis, S. Muthukrishnan, and M. J. Strauss. Surfing wavelets on streams: one-pass summaries for approximate aggregate queries. In Proc. 27th International Conf. on Very Large Data Bases (VLDB), pages 79-88, 2001.

[17] O. Goldreich. Combinatorial property testing (a survey). In In: Randomization Methods in Algorithm Design, pages 45-60. American Mathematical Society, 1998.

[18] P. Indyk and D. Woodruff. Tight lower bounds for the distinct elements problem. In Proc. 44th IEEE Symp. on Foundations of Computer Science (FOCS), page 283, 2003.

[19] E. Kushilevitz and N. Nisan. Communication Complexity. Cambridge University Press, Cambridge, UK, 1997.

[20] J. Misra and D. Gries. Finding repeated elements. Science of Computer Programming, 2:143152, November 1982.

[21] S. Muthukrishnan. Data Streams: Algorithms and Applications. Foundations and Trends in Theoretical Computer Science. Now Publishers, August 2005.

[22] A. Pavan and S. Tirthapura. Range-efficient counting of distinct elements in a massive data stream. SIAM Journal on Computing, 37(2):359-379, 2007. (Also in ICDE2005).

[23] D. Ron. Property testing: A learning theory perspective. Foundations and Trends in Machine Learning, 1(3):307-402, 2008.

[24] R. Rubinfeld and A. Shapira. Sublinear time algorithms. SIAM Journal on Discrete Mathematics, 25(4):1562-1588, 2011.

[25] N. Shrivastava, C. Buragohain, D. Agrawal, and S. Suri. Medians and beyond: new aggregation techniques for sensor networks. In SenSys, pages 239-249, 2004.

[26] B. Xu, S. Tirthapura, and C. Busch. Sketching asynchronous data streams over sliding windows. Distributed Computing, 20(5):359-374, 2008. 


\section{Appendix}

Additional figures plotting the processing time of both methods regarding the stream size over multiple data sets and different values for $\epsilon$. The time cost of GT's method is linear of the stream size, simply because their method processes every stream element, whereas our method's processing time is sublinear of the stream size. Also, our method overall is much faster than GT's, especially when the stream size (the number of 1-bits in the stream, indeed) becomes larger.

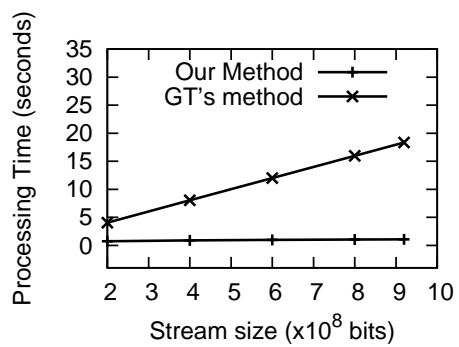

(a) Audio Bible

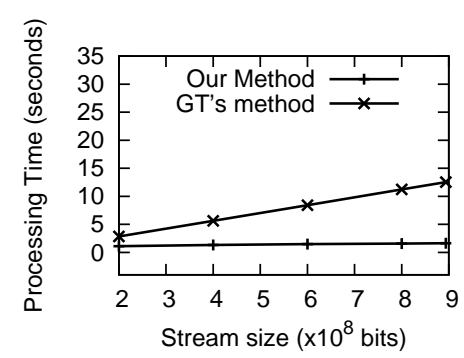

(d) Worldcup 98

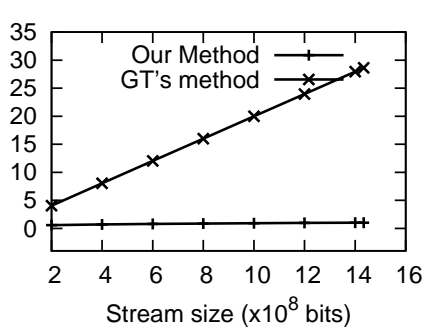

(b) Video of President

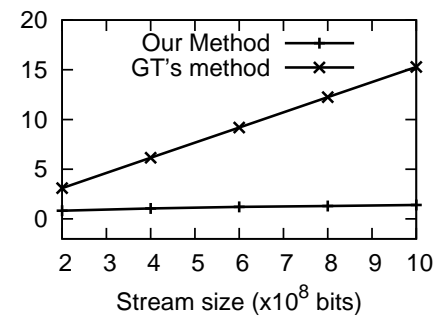

(e) Synthetic-0.3

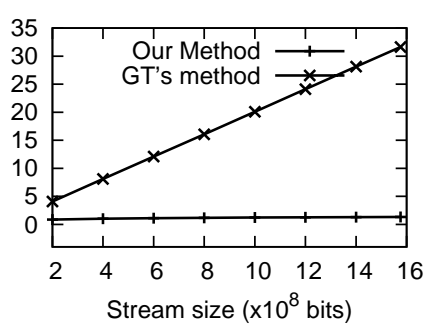

(c) Earth Image

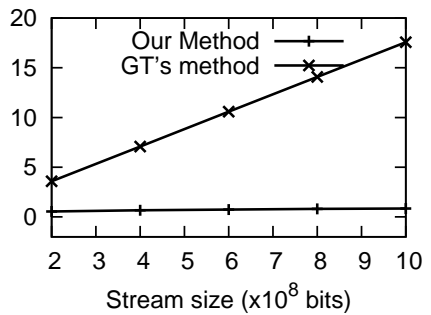

(f) Synthetic-0.4

Figure 5: Stream size vs. time, $\epsilon=0.02$ 


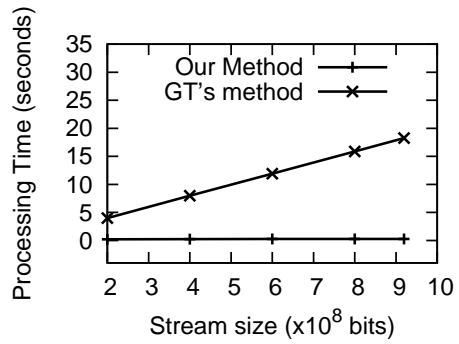

(a) Audio Bible

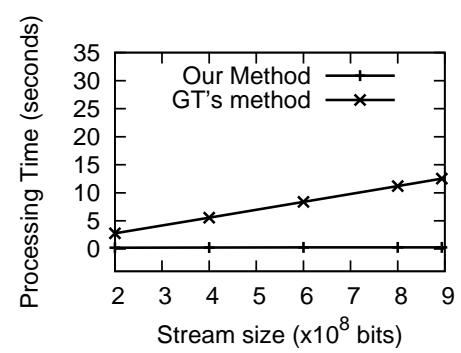

(d) Worldcup 98

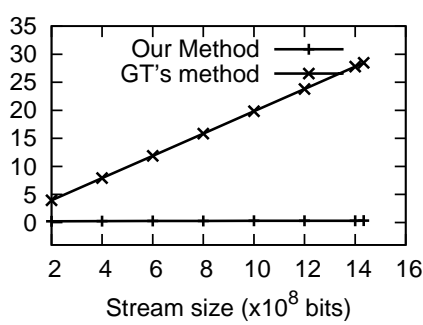

(b) Video of President

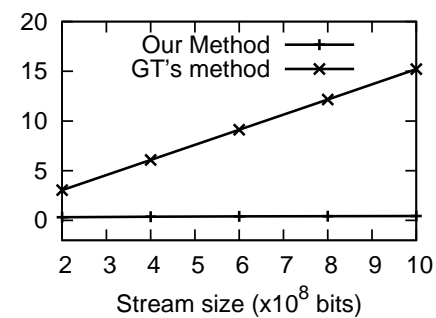

(e) Synthetic-0.3

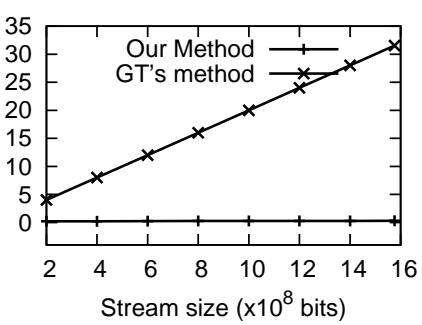

(c) Earth Image

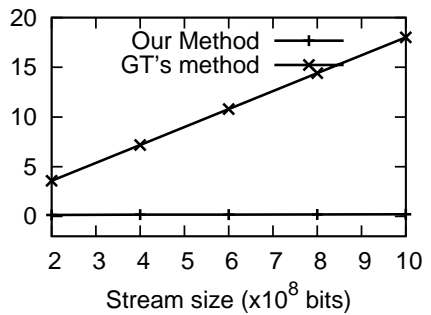

(f) Synthetic-0.4

Figure 6: Stream size vs. time, $\epsilon=0.05$

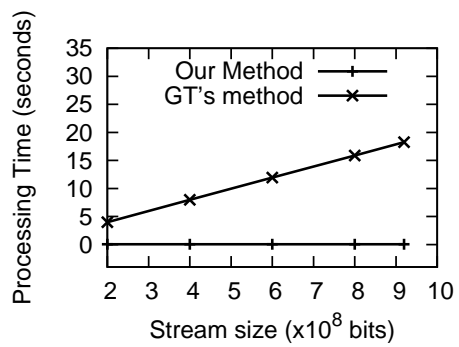

(a) Audio Bible

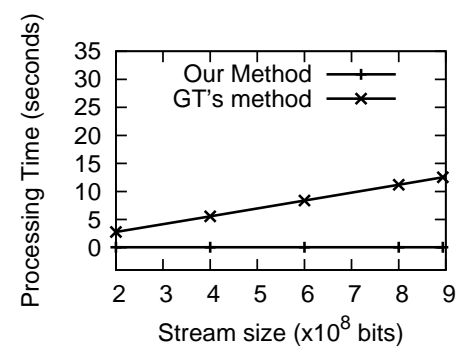

(d) Worldcup 98

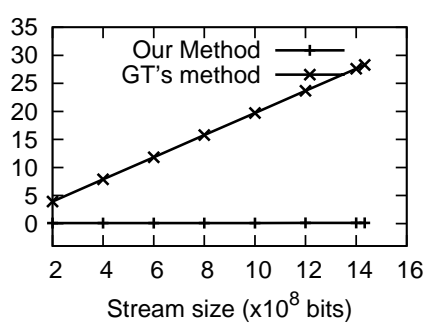

(b) Video of President

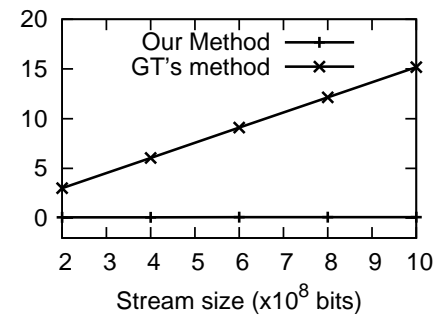

(e) Synthetic-0.3

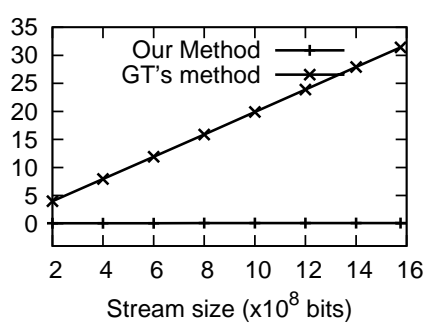

(c) Earth Image

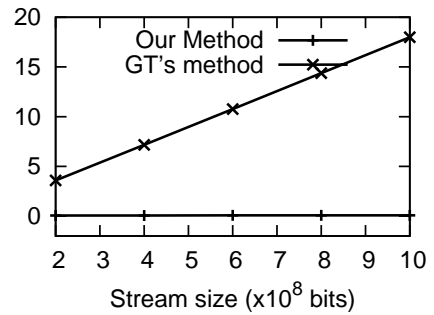

(f) Synthetic-0.4

Figure 7: Stream size vs. time, $\epsilon=0.1$ 


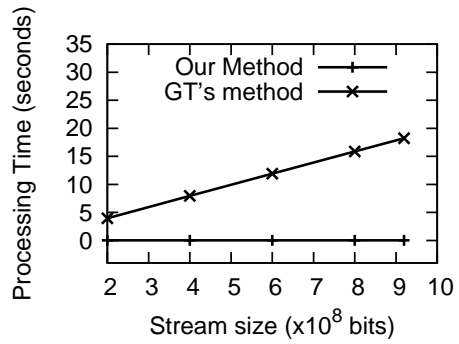

(a) Audio Bible

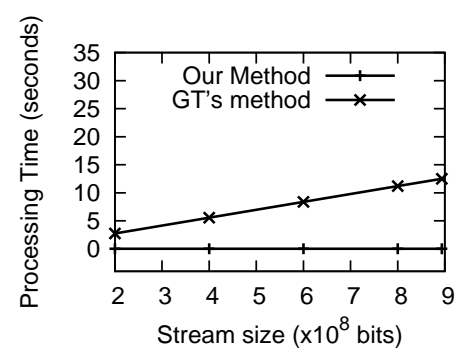

(d) Worldcup 98

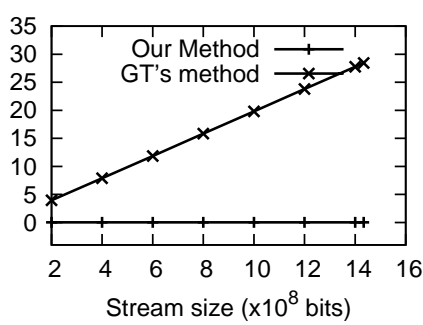

(b) Video of President

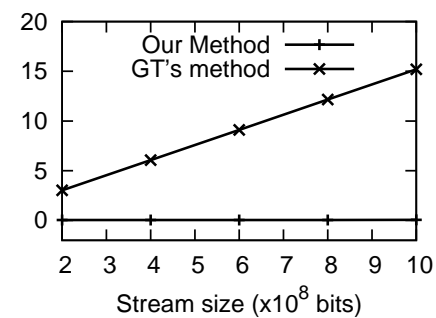

(e) Synthetic-0.3

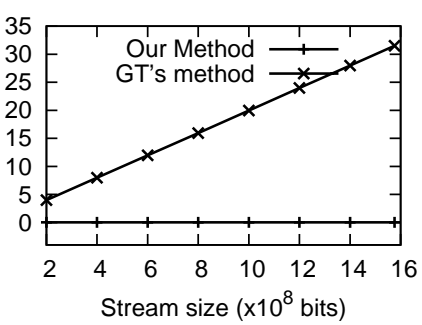

(c) Earth Image

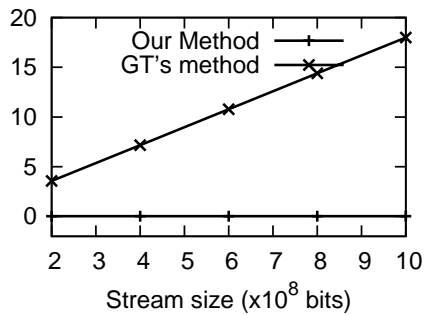

(f) Synthetic-0.4

Figure 8: Stream size vs. time, $\epsilon=0.2$

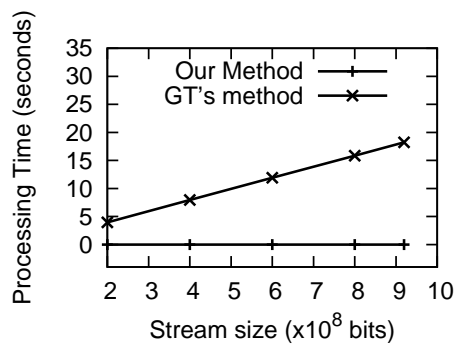

(a) Audio Bible

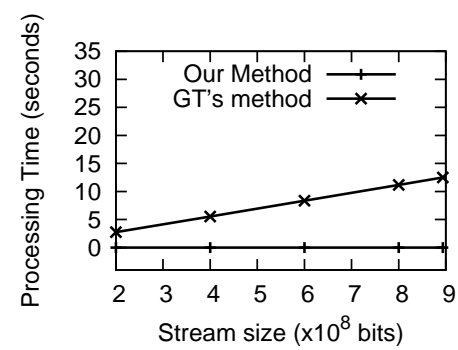

(d) Worldcup 98

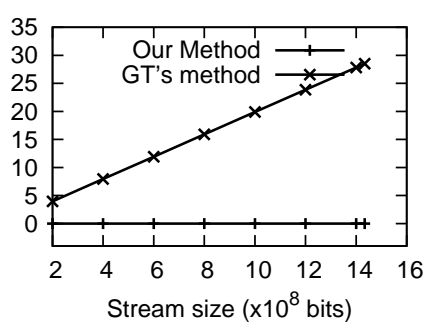

(b) Video of President

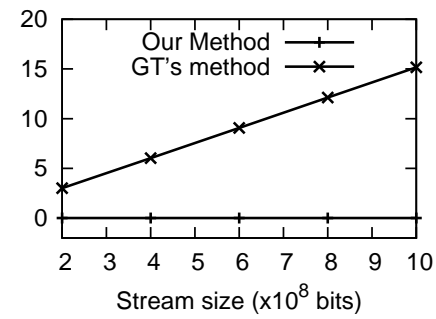

(e) Synthetic-0.3

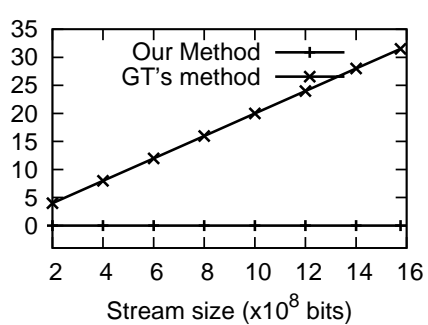

(c) Earth Image

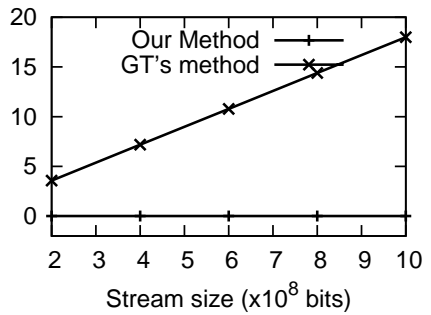

(f) Synthetic-0.4

Figure 9: Stream size vs. time, $\epsilon=0.5$ 